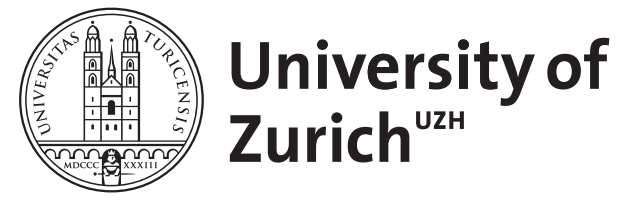

Zurich Open Repository and Archive

University of Zurich

University Library

Strickhofstrasse 39

CH-8057 Zurich

www.zora.uzh.ch

Year: 2012

\title{
Soziale Positionierungen im Kunstbetrieb. Linguistische Aspekte einer Soziologie der Kunstkommunikation
}

\author{
Hausendorf, Heiko
}

DOI: https://doi.org/10.1515/9783110278415.93

Posted at the Zurich Open Repository and Archive, University of Zurich ZORA URL: https://doi.org/10.5167/uzh-61383

Book Section

Published Version

Originally published at:

Hausendorf, Heiko (2012). Soziale Positionierungen im Kunstbetrieb. Linguistische Aspekte einer Soziologie der Kunstkommunikation. In: Müller, Marcus; Kluwe, Sandrar. Identitätsentwürfe in der Kunstkommunikation. Berlin, Germany; Boston, USA: Walter de Gruyter, 93-123.

DOI: https://doi.org/10.1515/9783110278415.93 
Heiko Hausendorf

\title{
Soziale Positionierungen im Kunstbetrieb
}

\author{
Linguistische Aspekte einer Soziologie der Kunstkommunikation
}

\section{Zur Einführung: „Alles andere fand ich einfach zum Kotzen“}

Einen komprimierten (und filmisch inszenierten) Eindruck von dem, wovon der vorliegende Beitrag handelt - von Kunstkommunikation und sozialen Positionierungen -, vermittelt der folgende Ausschnitt aus einem Film von Woody Allen (Manhattan, USA 1979), in dem sich die Protagonisten (Isaac und seine Freundin Tracy, Isaacs Freund Yale und seine Freundin Mary) zufällig treffen und anfangen, sich über die gerade gesehene Ausstellung auszutauschen:

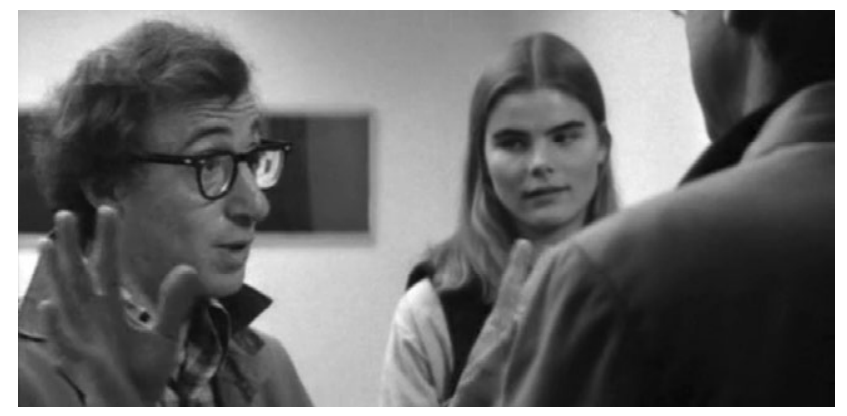

\section{Beispiel (1a): in der Castelli-Gallerie}

Isaac: Wir waren schon unten in der Castelli-Galerie und haben uns die Fotos angesehen - (beeindruckt) Unglaublich. Absolut unglaublich.

Tracy: Ja, ungeheuer.

Mary: (ungläubig, skeptisch) Die gefallen Ihnen also?

Isaac: $\quad$ Sie - äh - Sie meinen, die Fotografien da unten?

Mary: Ja, die da unten.

Isaac: (nachdrücklich) Klasse, absolute Klasse. Und Ihnen?

Mary: (Kopfschütteln, Schulterzucken) Nein, das kann ich leider nicht sagen. Für mich sind sie nur ein Plagiat - ein armseliger Abklatsch von Diane Arbus, aber ohne ihren Witz.

Isaac: $\quad$ Ach - äh - tatsächlich? Selbstverständlich gefielen sie mir nicht so wie die $(a b$ schätzig) Plexiglas-Skulpturen, aber ich meine, das war doch ... 
$\begin{array}{ll}\text { Mary: } & \text { (stirnrunzelnd) ... Ihnen gefiel dieses Plexiglas? } \\ \text { Isaac: } & \text { Gefielen Ihnen die Plexiglas-Skulpturen auch nicht? } \\ \text { Mary: } & \text { Aha, sehr interessant, ähm ... (Kopfschütteln) } \\ \text { Isaac: } & \text { Jedenfalls immer noch besser als diese - diese (belustigt) Stahlwürfel, die haben } \\ & \text { wir auch gesehen ... } \\ \text { Tracy: } & \text { Ja. } \\ \text { Mary: } & \text {... also die fand ich (bewundernd) grandios. Einfach grandios. } \\ \text { Isaac: } & \text { (ungläubig, irritiert) Die Stahlwürfel? } \\ \text { Mary: } & \text { Ja! Also für mich waren die unwahrscheinlich strukturell, ich meine, sie waren } \\ & \quad \text { vollkommen integriert. (nachdrücklich) Sie hatten - ähm - so eine fantastisch ne- } \\ & \text { gative Potenz. Alles andere fand ich einfach zum Kotzen! }\end{array}$

Auch wenn wir die Objekte, von denen hier die Rede ist (,Fotos“, „PlexiglasSkulpturen“ und „Stahlwürfel“) nicht gesehen haben (die Zuschauer des Films bekommen sie auch nicht zu sehen), verstehen wir mit, dass in diesem Ausschnitt von Kunstwerken bzw. von Objekten die Rede ist, die zumindest als Kunstwerke thematisiert werden. Worauf gründet dieses Verstehen? Da ist zunächst das, was wir in diesem gedruckten Artikel nur unzulänglich wiedergeben können, die Situierung einer Szene, die offenkundig in einem Ausstellungsraum spielt: An den Wänden des Raumes hängen Bilder, im Hintergrund sieht man Leute beim Betrachten der an den Wänden hängenden Bilder. Der Raum scheint Teil eines größeren Ausstellungsgebäudes zu sein, in dem sich „unten“ die „Castelli-Galerie“ befindet, in der die fraglichen Objekte ,,angesehen" wurden (und die es auch im wirklichen Leben gibt). Schon diese ersten Daten appellieren an unsere Vertrautheit mit der Praxis des Ausstellungsbesuchs und dem dafür typischen Austausch über das Gesehene als etwas, das eigens ,anzusehen“ ist und also einen speziellen Anschauungswert besitzt, der es lohnend macht, eine „Galerie“ zu besuchen. Hinzu kommt, dass im Ausschnitt wenig später von einer bekannten Künstlerin die Rede ist („Diane Arbus“) und dass auf eines der Objekte mit dem Ausdruck „Plexiglas-Skulptur“ referiert wird. Eine „Skulptur“ ist, wie uns schon Wikipedia verrät, ein „dreidimensionales Kunstwerk“. Aber das sind nur die oberflächlichen lexikalischen Indizien dafür, dass es in dieser kleinen Szene um Kommunikation über Kunstwerke geht, die uns als Teil der Alltagspraxis mehr oder weniger vertraut ist. Auf diese Alltagspraxis soll hier mit dem Ausdruck Kunstkommunikation referiert werden. Wir wollen darunter den Bereich der Kommunikation über Kunst und mit Kunst verstehen und festhalten, dass es in diesem Bereich Eigentümlichkeiten gibt, die kommunikativer Natur sind: das Verweilen vor einem Objekt in einer Ausstellung, das Zuerkennen von Anschauungswert und das Reden über das Gesehene. Wenden wir uns nun etwas genauer der Rede $\mathrm{zu}$.

Auch beim flüchtigen Verfolgen dieser Szene wird offenbar, dass für die Kommunikation über Kunst offenbar die Frage Wie hat es Dir / Euch gefallen? eine Art Zugzwang darstellt, den man bedienen muss, wann immer man sich anschickt, etwas Gesehenes in seiner Eigenschaft als Kunstwerk zu thematisieren: ,unglaublich. absolut unglaublich“" ist das Erste, was Isaac sagt, nachdem 
er das Gespräch auf die „Fotos“ „unten in der Castelli-Galerie“ gelenkt hat. Und das Letzte, was Mary zur Ausstellung sagt, ist: „Alles andere fand ich einfach zum Kotzen." Man muss kein Linguist sein, um in diesen Aussagen Erscheinungsformen des Bewertens zu erkennen. Wir wollen darunter zunächst nicht mehr als den Zugzwang verstehen, sich zur Frage des Gefallens (s. o.) irgendwie $\mathrm{zu}$ verhalten und damit diese Frage implizit oder explizit („Die gefallen Ihnen also?“) in ihrer pragmatischen Geltung zu bekräftigen. Wenn man der Szene glaubt, d. h. wenn man darin einen irgendwie charakteristischen Zug von Kunstkommunikation getroffen glaubt, kommt dem Bewerten für die Kunstkommunikation ein besonderer Stellenwert zu: Es ist der Fluchtpunkt, auf den das Ausstellen von Objekten, das Verweilen und das Anschauen sowie das Reden (oder Schreiben) darüber bezogen sind.

Und noch etwas ist in dieser Szene nicht leicht zu übersehen, wenn man speziell auf Marys Aussagen und ihre Mimik und Gestik achtet (beim Zuschauen): Einher mit dem Bewerten geht eine bestimmte Art der Selbstdarstellung, die dadurch geprägt ist, dass die Sprecherin ein (vernichtendes) Urteil über den ästhetischen Rang des Gesehenen fällt (negativ: „ein fader Abklatsch von [...]“; positiv: „eine phantastisch negative Potenz“). Es ist entsprechend eine Selbstdarstellung als Kunstrichter(in) bzw. -kritikerin, der es zukommt, über Rang und Wert von Kunst zu entscheiden. Wir wollen das als Ausdruck einer bestimmten sozialen Position und die Art und Weise der Inanspruchnahme dieser Position beim und durch das Reden als soziale Positionierung verstehen. Kunstkommunikation, so die Botschaft dieser kleinen Szene, ist neben allem anderen, was es noch sein mag, auch und gerade ein Schauplatz für soziale Positionierungen, mit denen die Teilnehmenden sich wechselseitig aufzeigen, wer oder was sie sind bzw. als wer oder was sie betrachtet und verstanden werden wollen. Auch dazu muss man die Szene nicht linguistisch analysieren. Im Film selbst wird die fragliche soziale Position regelrecht inszeniert; dazu trägt neben Mimik, Gestik und Prosodie insbesondere der spezielle Jargon der Kunstkritik bei, der hier durch den Kunstgriff einer leichten Übertreibung und Verzerrung der Belustigung anheimgestellt wird (,unwahrscheinlich strukturell“; ,vollkommen integriert", „fantastisch negative Potenz"). Wenig später im Film wird sich Isaac im Gespräch mit seiner Freundin Tracy über das ,„pseudointellektuelle Geschwätz“ von Mary aufregen. Es kann also kein Zweifel bestehen, dass es hier auch und gerade darum geht, ein bestimmtes Image vorzuführen und zu karikieren - mit dem Effekt einer speziellen Art der Komik.

Nicht immer ist der Alltag der Kunstkommunikation so komisch. Aber dass es bei Kunstkommunikation immer auch um das Einnehmen einer bestimmten sozialen Position geht und dass sich diese Positionierung typischerweise im Rahmen des Bewertens abspielt, scheint durchaus generalisierbar. Zumal dieser Gedanke eine historische Tiefenschärfe hat, die bis in das Aufkommen der bürgerlichen Gesellschaft zurück reicht: Seit Habermas bahnbrechenden Untersuchungen, die er unter dem Titel „Strukturwandel der Öffentlichkeit“ 1962 erstmals publiziert hat (Habermas 2006), kann man wissen, dass die Kommunikation über Kunst und speziell das Ausbilden eines Kunstge- 
schmacks die soziopragmatischen Kontexte liefern, in denen sich Personen, Autoren und Autorinnen, aber auch Zuhörer und Zuhörerinnen, Leser und Leserinnen, also ein Publikum, als „Bürger“ wahrzunehmen beginnen. Die Ausbildung einer für das Selbstverständnis der modernen Gesellschaft zentralen Kategorie der Selbst- und Fremdwahrnehmung läuft also, vereinfacht gesagt, auch und gerade über das Bewerten als Teil einer sich entwickelnden kommunikativen Praxis der Kunstkommunikation und den damit sich manifestierenden sozialen Positionierungen. Es ist also kein Zufall, wenn in dem oben wiedergegebenen Filmausschnitt das Reden über Kunst und entsprechend ein Ausstellungsraum als Bühne gewählt werden, um eine für ein bestimmtes soziales Milieu charakteristische Szene vorzuführen, die ihre gesellschaftliche Selbstvergewisserung durch die Markierung „feiner Unterschiede“ (Bourdieu 1982) im Kunstgeschmack betreibt. Die nächste Einstellung im Film zeigt uns Mary und ihren Freund Yale, wie sie auf dem Weg zu einem Restaurantbesuch mit Isaac und Mary dabei sind, eine Liste der ,überschätzten“ Künstler zusammen zu stellen (die u. a. Ingmar Bergmann, Vincent van Gogh, Gustav Mahler, F. Scott Fitzgerald und Heinrich Böll enthält).

Nicht nur die Eigennamen dieser Künstler verweisen auf entsprechende „Namenkennergruppen“ (von Polenz 1988: 122), auch die Art und Weise, wie Mary den Namen „,van Gogh“ ausspricht (mit stilisiert pharyngalem ,ach“Laut), ist vor allem ein Akt der sozialen Positionierung, mit dem sich die Sprecherin als Kennerin präsentiert. Und genau dieser „Akt des Sprechens“ (Bourdieu 1990) wird dann wenig später im Klatsch mit Tracy von Isaac karikierend aufgenommen, um sich über diese Art der sozialen Selbstpositionierung zu empören und darauf selbst mit einer ausgebauten Fremdtypisierung zu antworten:

Beispiel (1b): nach der Ausstellung

Isaac: Ich weiss nicht, woher dieses kleine (betont) Ratcliffe-Scheusal die Frechheit hernimmt, Leute wie Scott Fitzgerald und Gustav Mahler und Heinrich Böll -

Tracy: (unterbricht) Ich weiß nicht, wieso Du Dich so aufregst?

Isaac: Ich reg mich auf, weil mich dieser pseudo-intellektuelle Mist auf die Palme bringt. (ahmt Sprechweise nach) ,van Gach“, hast Du gehört, wie sie das gesagt hat? (wiederholt) „van Gach“: wien Araber.

[...]

Isaac: ((über Yale)) hat eine zauberhafte Frau und läuft dieser dieser (zögert) dieser (verächtlich) entsetzlichen College-Ziege nach. Er ist aber schon immer auf diese Sorte reingefallen. Auf Frauen, die wie eine tibetanische Gebetsmühle unentwegt von (zitiert) existentieller Realität quasseln. Die immer auf dem Fußboden hocken mit Wein und Käse und alles zerreden. Und Worte wie allegorisch und didaktisch falsch aussprechen.

Der Zusammenhang von Kunstkommunikation und sozialen Positionierungen ist vor allem in der Soziologie gut bekannt. Weniger bekannt sind allerdings die sprachlichen Erscheinungsformen, in denen sich dieser Zusammenhang alltäglich manifestiert. Während z. B. das Bewerten für sich genommen, d. h. 
außerhalb bestimmter gesellschaftlicher Funktionsbereiche, linguistisch gut untersucht ist und die sprachlichen Mittel und Formen sozialer Positionierungen und sozialer Kategorisierungen gerade in den letzten rund 20 Jahren sehr viel konversations- und diskurslinguistische Aufmerksamkeit gefunden haben, ist die Kunstkommunikation in der Linguistik nach wie vor eine tabula rasa. Man sieht das auf den ersten Blick, wenn man die gesprächs- und textlinguistische Erforschung von Kunstkommunikation mit der von Rechts-, Gesundheits-, Wirtschafts- und politischer Kommunikation vergleicht. In den einschlägigen gesprächs- und textlinguistischen Handbüchern wird „Kunst“ gerade erst ein relevantes Stichwort (vgl. z. B. die beiden Halbbände zur Text- und Gesprächslinguistik, Brinker et al. 2000; Brinker et al. 2001, in denen Kunstkommunikation als eigenständiger Kommunikationsbereich noch gar nicht vorkommt). Auch wenn sich dieser Forschungsstand allmählich zu verändern beginnt (vgl. z. B. die Beiträge in Hausendorf 2007b), springt sofort ins Auge, dass sich bisherige Untersuchungen in diesem Bereich fast ausschließlich auf den Ausschnitt der öffentlichen Kunstkritik mit ihren einschlägigen Textsorten konzentriert haben. Dabei ist i. d. R. nicht nur der größere Zusammenhang der Kunstkommunikation vernachlässigt worden, der eben mehr als nur das Bewerten umfasst. Bezeichnenderweise ist auch der Zusammenhang der sozialen Positionierung weitgehend ausgeblendet worden (eine textlinguistische Ausnahme ist die Arbeit von Christiane Thim-Mabrey, Thim-Mabrey 2001; eine gesprächslinguistische Ausnahme ist die frühe Arbeit von Walther Kindt, Kindt 1982). Die etwa im Hinblick auf jenen oben illustrierten charakteristischen Jargon der Kunstkritik unmittelbar einschlägigen Arbeiten stammen nicht aus der Linguistik, sondern z. B. aus der Kunstkritik selbst (Smolik 2001) oder z. B. aus der Kunstgeschichte (Demand 2003; Ullrich 2003).

In beiden Hinsichten, mit Blick auf die Praxis der Kunstkommunikation und mit Blick auf die damit einhergehenden sozialen Positionierungen, besteht also Nachholbedarf. Der vorliegende Beitrag setzt an dieser Stelle an, indem er ein linguistisches Modell der Kunstkommunikation aufgreift und ausgehend von diesem Modell nach charakteristischen sozialen Positionen und Positionierungen im Kunstbetrieb fragt.

Es sei vorausgeschickt, dass die folgenden Bemerkungen ein etwas lockeres Verhältnis zur Empirie haben, das mit den Gewohnheiten der Gesprächsund Textlinguistik nicht leicht $\mathrm{zu}$ vereinbaren ist. Es geht mir eher darum, Perspektiven für empirische Studien aufzuzeigen als darum, selbst eine geschlossene empirische Studie an dieser Stelle zu präsentieren. Natürlich muss man sich, wenn man nicht den Boden unter den Füßen verlieren möchte, mit Beispielen verständlich machen. Ich greife dazu auf das zurück, was aus früheren eigenen Studien vorliegt und was speziell eine für die Zwecke dieses Artikels in Angriff genommene Lektüre erbracht hat. Das ist die Lektüre eines Katalogs, der kürzlich anlässlich einer Seurat-Ausstellung im Kunsthaus Zürich entstanden und publiziert worden ist; daraus speziell die Texte von Wilhelm Genazino und von Gottfried Boehm. Die Auswahl ist willkürlich, aber wie immer nicht zufällig. Beide Texte finden sich im Ausstellungskatalog und 
sind damit Bestandteil eines besonders prominenten Segments von Kunstkommunikation (s. u. 2).

\section{Kommunikative Aufgaben und soziale Positionen vor dem Kunstwerk}

Roland Barthes hat in einem (zuerst 1979 erschienenen) Beitrag über Cy Twombly (Barthes 1983) eher beiläufig von einer „Ästhetik“ gesprochen, ,die nicht das Werk an sich studiert, sondern das Werk, wie es der Betrachter oder der Leser in sich selber zum Sprechen bringt: eine Typologie der Diskurse gewissermaßen“ (S. 89). Eine solche Typologie hätte die „Subjekte“ bzw. „Subjekttypen“ zum Gegenstand, „die das Bild betrachten“ (ebd.) und vornehmlich durch ihr Sprechen, durch den „Diskurs“, „,en sie vor dem betrachteten Gegenstand halten“, konstituiert werden (ebd.). Barthes führt dann illustrierend einige dieser „Subjekte“ an, von denen wir hier die ersten drei wiedergeben wollen: ${ }^{1}$

\footnotetext{
„Es gibt das Subjekt der Kultur, der Bildung, das weiß, wie Venus geboren ist, wer Poussin oder Valéry sind; dieses Subjekt ist beredt, es kann überströmend sprechen. Es gibt das Subjekt der Spezialisierung, das die Geschichte der Malerei gut kennt und über den Platz, den Twombly darin einnimmt, zu reden weiß. Es gibt das Subjekt des Vergnügens, das sich vor dem Bild erfreut, das bei seinem Erblicken ein Jubilieren verspürt, ohne es so recht aussprechen zu können; dieses Subjekt ist also stumm; es vermöchte nur auszurufen: „Wie schön das ist!" und das zu wiederholen; es ist das eine der kleinen Qualen des Sprechens: man kann nie erklären, warum man diese oder jene Sache schön findet: die Lust erzeugt eine Art Sprechfaulheit, und wenn man von einem Werk sprechen will, muss man den Ausdruck des Genusses durch abgelegenere, rationalere Reden ersetzen [...].“ (S. 89 f.)
}

Barthes hat die hier angedeutete „Typologie“ meines Wissens weder in seinen Arbeiten zu C. T. noch anderenorts weiter ausgeführt, und speziell bei den hier nicht wiedergegebenen „Subjekten der Erinnerung“ und der „Produktion“ verliert sich mehr und mehr der Zusammenhang zum „Diskurs“. Es bleibt auch unklar, was genau der empirische Status dieser „Subjekte“ ist. Die „Typologie" liest sich auch wie eine Reflexion der eigenen Zugangsweisen zum Werk Twomblys.

Und doch versteckt sich in diesen Bemerkungen $\mathrm{m}$. E. ein viel versprechendes Forschungsprogramm, das der Leitidee des vorliegenden Beitrags sehr nahe kommt. So kann man ohne viel Mühe im Sprechen von Isaac und Tracy das „Subjekt des Vergnügens“ manifestiert sehen (,unglaublich. absolut unglaublich“), und im Sprechen von Mary ein Subjekt, das ,überströmend sprechen“ kann, sicher ein „Subjekt der Spezialisierung“ und vielleicht auch ein Subjekt des richtenden Urteils. Wie auch immer: Da ist zunächst die Umfokussierung auf den, der vor dem Kunstwerk steht und einen „Diskurs hält“ und der durch diesen Diskurs konstituiert wird (,ein Subjekt ist [...] immer durch

1 Die Erläuterungen der „Subjekte“ beziehen sich auf die Bilder von Twombly, sie sind aber leicht zu verallgemeinern. 
sein Sprechen konstituiert“; S. 89). Wir übersetzen diesen Gedanken in die Orientierung an einem Gegenstand der Kunstkommunikation (vereinfacht gesagt: Sprechen und Schreiben über Kunst) ${ }^{2}$ und in die Frage nach der Phänomenologie des Redens und Schreibens über Kunstwerke. Wir wollen, um in dieser Richtung weiter zu kommen, mit der Konversationsanalyse davon ausgehen, dass man Erscheinungsformen der Kommunikation über Kunst als Antworten auf zugrunde liegende Fragen verstehen kann und dass man diese Fragen rekonstruierend aus den Antworten zu entdecken hat. Ziel ist also nicht, sich vorgängig zu überlegen, worum es in der Kunstkommunikation geht (Was sind die wesentlichen Fragen?), sondern Kunstkommunikation in ihren empirischen Erscheinungsformen zu dokumentieren und als eine Praxis zu rekonstruieren, in und mit der kommunikative Probleme bzw. Aufgaben gelöst werden.

Welches sind nun die kommunikativen Aufgaben, die diese Kommunikationspraxis auszeichnen? Wir wollen dazu auf Vorarbeiten zu einem Beschreibungsmodell zurück greifen, die es berechtigt erscheinen lassen, von insgesamt fünf zentralen kommunikativen Aufgaben auszugehen, die zusammen das ausmachen, was man als das Grundgerïst der Problemstellungen ansehen könnte, die mit dem Sprechen und Schreiben über Kunst offenbar verbunden sind (Hausendorf 2005; Hausendorf 2006; Hausendorf 2007a). Dabei handelt es sich um das Bezugnehmen, das Beschreiben, das Deuten, das Erläutern und das Bewerten. Auch wenn es durch die Verwendung von Verben so klingen mag: Damit sind nicht „Sprechakte“ gemeint, sondern kommunikative Aufgaben, mit denen eine spezifische Frage- und Problemstellung verbunden ist, die es kommunikativ irgendwie zu bearbeiten gilt (s. u. Fig. 1). Das ist die erste Ebene des Modells, und auf ihr ist noch gar nicht $\mathrm{zu}$ entscheiden, wie und durch wen die Aufgaben bearbeitet werden. Diese Frage stellt sich erst auf der nächsten Beschreibungsebene der sogenannten Mittel: Die Mittel sind die Verfahren und Strategien, in denen sich die Pragmatik und die Semantik der Aufgabenerledigung erfassen lässt. Auf dieser zweiten Beschreibungsebene kann man dann von konkreten Sprechakten sprechen (wenn man das will). Wie schließlich die sprachlichen Erscheinungsformen aussehen, mit denen die Mittel im Text bzw. im Gespräch ausgedrückt und manifestiert werden, ist auf der dritten Beschreibungsebene zu erfassen, sie hat es mit dem Lexikon und der Grammatik der jeweiligen (einzel)sprachlichen Formen zu tun. Die folgende Übersicht soll diese dreigliedrige Struktur veranschaulichen (s. Abb. 1). Die ausgewählten Mittel und Formen werden im vorliegenden Beitrag nach und nach eingeführt.

Aufgaben, Mittel und Formen sind in einem strikten Sinn phänomenologischer Natur: Mit ihnen wird das nachgezeichnet, was sich in den Texten und

2 Genau genommen erschöpft sich Kunstkommunikation nicht in der Kommunikation über Kunst, sondern schließt auch die Kommunikation mit und durch Kunst ein (Luhmann 1997). Man kann auch sagen: es geht darum, wie in der Kommunikation über Kunst Kommunikation mit und durch Kunst möglich und wahrscheinlich gemacht wird (vgl. dazu Hausendorf 2006). 
Gesprächen über Kunstwerke immer wieder als empirische Antwort auf die zugrunde liegende(n) Frage(n) findet. Weder geht es dabei um normative Vorstellungen von dem, was zum Sprechen und Schreiben über Kunst dazu gehören sollte, noch beanspruchen die Aufgaben und die Mittel eine irgendwie geartete theoretische Ableitung. Gezeigt und illustriert werden soll vielmehr, dass und wie sich das Sprechen und Schreiben über Kunst als eine Praxis des Bezugnehmens, Beschreibens, Deutens, Erläuterns und Bewertens empirisch rekonstruieren lässt.

Auf der allgemeinen Beschreibungsebene des Modells wird Vollständigkeit angestrebt. Die genannten Aufgaben sollen die für Kunstkommunikation konstitutiven Problemstellungen abbilden. Schon auf der Mittel-Ebene sind die Befunde nicht mehr so leicht überschaubar. Für jede der genannten Aufgaben gibt es eine Reihe verschiedener Mittel, von denen hier nur jeweils ein Mittel herausgegriffen wurde. Weitere Mittel finden sich in den o. g. Vorarbeiten. Auf der Formenebene schließlich ergeben sich mit jeder neuen Probe naturgemäß auch neue Eintragungen. Linguistisch gesehen geht es dann darum, diese Formen grammatisch und lexikalisch zu analysieren, so dass hervortritt, welche Aspekte der sprachlichen Formgebung für die Realisierung eines bestimmten Mittels zur Lösung einer bestimmten Aufgabe kommunikativ ausgenutzt werden können. ${ }^{3}$

Wir können mit Hilfe eines solchen Modells etwas genauer angeben, welcher Art die „Diskurse“ sind, von denen Barthes spricht (s. o.): Der „Diskurs“, das ist ja zunächst das Gesprochene, das man findet, wenn man Leute dabei beobachtet, wie sie sich vor einem Kunstwerk verhalten und unterhalten. Und was man hier findet, das ist - in unzähligen Variationen und in ganz unterschiedlicher Ausprägung - das Bezugnehmen auf ein Werk, das Beschreiben, das Deuten, das Erläutern und das Bewerten eines Werkes.

In diesen Sprachspielen des Bezugnehmens, Beschreibens, Deutens, Erläuterns und Bewertens manifestieren sich zugleich und weiterführend typische Sprechweisen und Kombinationen von Sprechweisen, die man immer wieder findet und die man zum Gegenstand einer „Typologie“ machen kann. Barthes spricht dazu von einer „Typologie der Diskurse“, die für ihn gleichbedeutend ist mit einer Typologie der „Subjekte“ (s. o.). Wir ziehen es vor, an dieser Stelle von sozialen Positionen zu sprechen und wollen damit hervorheben, dass sich Sprechweisen zugunsten erwartbarer kommunikativer Strukturen verfestigen und verselbständigen, auf die man im Sinne einer charakteristischen Position referieren kann. Musterhaft ausgeprägte Sprechweisen machen es möglich, dass Sprecher und Sprecherinnen positionsbezogen zugeordnet, dass positionsspezifisch Eigenschaften und Verhaltensweisen zugeschrieben und dass Personen positionsspezifisch bewertet werden können. Sprecher und Sprecherinnen erscheinen dann als das oder das bzw. der oder die (Hausendorf 2000). Die Frage, wer spricht, die sich immer stellt, wird also nicht individuell,

3 Anders als es die modellorientierte Darstellung suggerieren mag, ist diese Art von Analysearbeit keineswegs abgeschlossen. Es handelt sich vielmehr um ein work in progress, das im Prinzip auf jeder Ebene und an jeder Stelle empirisch weiter zu entwickeln ist. 


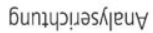

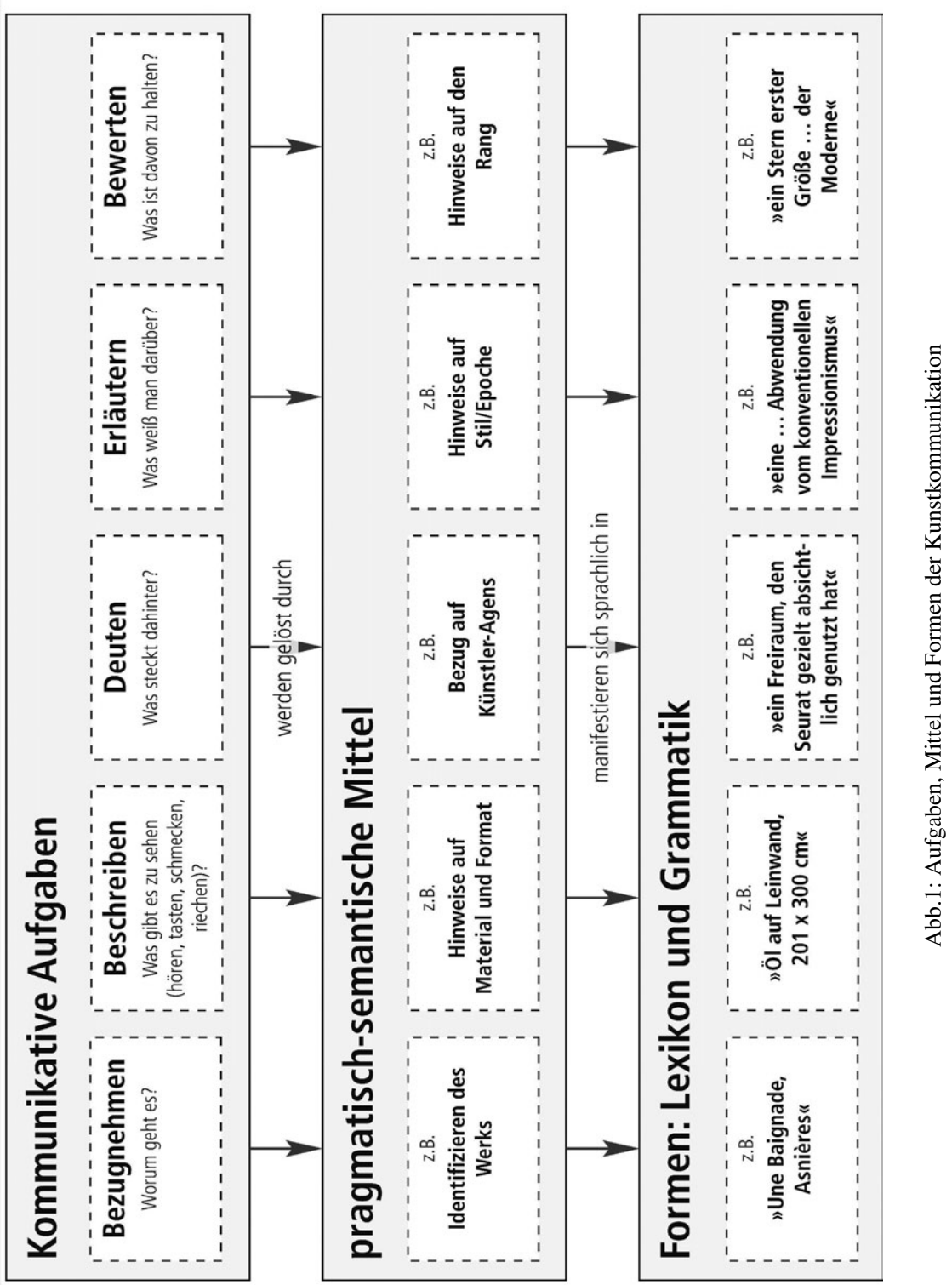


sondern sozial beantwortet: es ist eine soziale Position, die sich im Sprechen über Kunst konstituiert. Sie jedenfalls ist es, die im vorliegenden Beitrag interessiert.

Wenn man soziale Positionierungen untersuchen will, muss man versuchen, die oben skizzierten Mittel und Formen des Bezugnehmens, Beschreibens, Deutens, Erläuterns und Bewertens auf ihr Verhältnis zu sozialen Positionen im Kunstbetrieb zu befragen. Man muss, anders gesagt, die Mittel und Formen zugleich als Beiträge zu sozialen Positionierungen verstehen, die in der Kunstkommunikation immer wieder auftauchen und insgesamt die gesellschaftliche Struktur des Kunstbetriebes ausmachen. Auch das ist zuerst eine empirische Frage, geht es doch darum, anhand typischer wiederkehrender Sprech- und Schreibereignisse nach den kommunikativen Strukturen zu fragen, die für solche Ereignisse konstitutiv sind. Dahinter steht ein Verständnis von sozialen Positionen, das diese als Verfestigungen und Erstarrungen kommunikativer Erwartungen begreift, die sich losgelöst von Personen aus Fleisch und Blut („Akteuren“) kommunikativ verselbständigt haben und unterschiedliche Formen sozialer Kategorisierung annehmen können (vgl. dazu auch Hausendorf/Bora 2006). Aus unserem alltäglichen Vertrautsein mit dem Kunstbetrieb ${ }^{4}$ mögen uns Kandidaten für solche Positionen mehr oder weniger vertraut sein: als Besucher von Ausstellungen, in denen Künstler vermittelt durch Ausstellungsmacher, Kuratoren und Sammler ihre Werke ausstellen, auf die in Katalogen und Begleittexten durch Kunstwissenschaftler Bezug genommen wird und die in der Ausstellung durch Kunstführer und -pädagogen einem Publikum nahegebracht werden. $\mathrm{Ob}$ und welche Mittel und Formen der kommunikativen Aufgaben der Kunstkommunikation mit diesen Leerstellen verknüpft sind, ist damit natürlich noch nicht gesagt und erst recht nicht empirisch gezeigt. Dazu bedarf es der empirischen Analyse konkreter Erscheinungsformen - Z. B. im Rahmen des Ausstellens von Kunstwerken, dem im modernen Kunstbetrieb eine Schlüsselrolle zukommt (vgl. Wulffen 1994; Kravagna 2001; Huber et al. 2002; Dröge/Hoffmann 2010; Ziese 2010).

$\mathrm{Zu}$ klären ist dabei, ob und in welcher Weise mit den oben genannten Aufgaben, ihren Mitteln und ihren Formen typischerweise bestimmte soziale Positionierungen einhergehen. Wenn man unbefangen genug herangeht, betrifft das bereits die Rollenverteilung von „Künstler“ und „Betrachter“: „Auf je zwei bildende Künstler", zitiert Christian Demand den Bildhauer Jürgen Weber, „entfällt eine Kraft, die dafür bezahlt wird, dass sie über Kunst spricht. In den meisten Fällen wird sie sogar besser bezahlt, als die beiden Künstler" (Demand 2007, S. 265). Schon das Reden über Kunst ist also alles andere als „unschul-

4 Wir verwenden den Ausdruck „Kunstbetrieb“ in diesem Beitrag, um darauf aufmerksam zu machen, dass es einen gesellschaftlich ausdifferenzierten Kommunikationsbereich gibt, mit dem sich in der Neuzeit allmählich ein auf das Erleben und Behandeln von Welt als Kunst spezialisiertes, mehr oder weniger autonomes gesellschaftliches Teilsystem mit eigenen Organisationen und Institutionen entwickelt hat, so dass Kunstkommunikation gleichrangig neben andere spezialisierte Formen wie die politische, die Wirtschafts-, Glaubens-, Gesundheits- oder Rechtskommunikation tritt (vgl. dazu Lüddemann 2007). 
dig“, sondern bereits Ausdruck einer bestimmten sozialen Positionierung als Teil des Kunstbetriebs. Wer Kunstkommunikation phänomenologisch erfassen möchte, darf von dieser ihrer Einbettung in den Kunstbetrieb nicht schweigen. Für die Aufgabe des Bewertens ist ein solcher Zusammenhang von Kunstkommunikation und sozialen Positionierungen, wie eingangs schon illustriert, mit der Kunstkritik gesellschaftlich institutionalisiert und entsprechend gut zu belegen. Der Künstler ist i. d. R. nicht sein eigener Kunstkritiker. Wir werden darauf am Ende des Artikels wieder zurück kommen, zuvor aber der Reihe nach die anderen Aufgaben der Kunstkommunikation und ihre Implikationen für soziale Positionierungen durchgehen. Dabei kann von vornherein kein Anspruch auf Vollständigkeit und gut durchdachte Systematik erhoben werden. Vielmehr geht es um den Versuch, deutlich zu machen, wie sich in unscheinbaren sprachlichen Mitteln und Formen des Redens und Schreibens über Kunstwerke grundlegende soziale Positionierungen des Kunstbetriebs manifestieren.

Der dies schreibt, ist Sprachwissenschaftler, kein Kunstsoziologe und erst recht kein Kunsthistoriker. Die Aufmerksamkeit gilt der Pragmatik und Semantik der Sprache(n) der Kunstkommunikation mehr als dem Kunstbetrieb, innerhalb dessen die sozialen Positionierungen, von denen gleich die Rede sein wird, (kunst)soziologisch und (kunst)historisch zu verankern und zu entwickeln wären. Dazu können im Folgenden nur ein paar kursorische Anmerkungen gemacht werden, die ausgewählte Querverbindungen andeuten sollen.

\section{Bezugnehmen}

Die Kommunikation über Kunstwerke, die mündliche wie die schriftliche, hat eine Implikation, die man ob ihrer vermeintlichen Selbstverständlichkeit leicht übersieht. Sie verlangt, dass irgendwie klargestellt wird, wovon überhaupt die Rede ist. Es ist das „es“ in einer Frage wie „Hat es Dir/Euch gefallen?“, das im Reden wie im Schreiben über Kunst impliziert ist und einer Auflösung bedarf. Wir wollen hier von einer Aufgabe des Bezugnehmens sprechen: In und mit Kommunikation muss sichergestellt werden, worauf genau Bezug genommen wird, wenn etwas als Kunstwerk erlebt und behandelt werden soll. Wenn man nach der Art der empirischen Auflösung dieser Aufgabe fragt, scheiden sich die Wege der mündlichen und der schriftlichen Kunstkommunikation. Werfen wir zunächst einen Blick auf die Mündlichkeit. Wenn wir an ein Gespräch in einer Ausstellung denken, wie im eingangs zitierten Filmbeispiel, wird sofort evident, was alles dafür getan werden kann, damit klar wird, worum es geht. Die „Fotos“, über die im Ausschnitt aus dem Film geredet wird, sind ja nicht einfach „da“, sondern ihr „Da-Sein“ wird demonstriert und präsentiert, wird sozusagen unübersehbar gemacht, wird vorgezeigt (in der "Castelli-Galerie“). Genau darin liegt wohl die kommunikative Kraft des Ausstellens: zu zeigen, worum es gehen soll für die Besucher. Nicht zufällig hat man vom „Zeigegestus" des Ausstellens gesprochen (Locher 2002: 21). Unter Anwesenden wird dieser kommunikative Vorlauf bereits in dem Moment interaktiv relevant 
gesetzt, in dem sich die Beteiligten vor einem Werk versammeln und auf diese Weise ihre Wahrnehmung eines bestimmten Objektes für alle anderen Beteiligten wechselseitig wahrnehmbar machen. Dabei muss kein Wort gesprochen werden. Das „Zur-Schau-Stellen fokussierter Wahrnehmung“ (Hausendorf 2007a) ist selber bereits ein interaktiver Zug, an den auf vielfältige Weise angeknüpft und der auf vielfältige Weise differenziert werden kann. ${ }^{5}$ Es ist eine denkbar ökonomische, ebenso unauffällige wie effektive Weise, die Aufgabe des Bezugnehmens empirisch zu lösen. Interessanterweise geht mit dieser Lösung der Aufgabe im wahrsten Sinne des Wortes eine Position einher, die Position vor dem Kunstwerk. In dieser räumlichen Positionierung manifestiert sich eine für die Kunstkommunikation außerordentlich charakteristische soziale Positionierung: die Positionierung als Kunstbetrachter ${ }^{6}$. Sie ist viel weniger selbstverständlich, als man vielleicht annehmen mag. Man muss dazu nur an die Imperative denken, die mit dieser Positionierung verbunden sind (Nicht berühren!), an den Aufwand, der getrieben wird, diese Position auch räumlich zu begrenzen (Näherungs- und Betretbarkeitstabus) oder an die Verhaltenserwartungen, die auf den zukommen, der diese Position einnimmt (Stehenbleiben, Verweilen und Betrachten!). „Ein bisschen dumm“ hat Wolfgang Ullrich (Ullrich 2007) im Anschluss an einen berühmten Essay von Brian O'Doherty den (idealen) KUNSTBETRACHTER genannt:

„Wer ist dieser Betrachter? Manchmal Beschauer, Zuschauer, Besucher genannt. Er hat kein Gesicht, er hat zu allermeist nur einen Rücken. Er beugt sich vor und schaut genau hin und verhält sich etwas ungeschickt. Seine Haltung ist fragend, seine Verwirrung diskret. Er - und ich glaube, er ist mehr männlichen als weiblichen Geschlechts - trat zusammen mit der Moderne auf, d. h. mit dem Verschwinden der Perspektive. Er wirkt wie eine Ausgeburt des Bildes und wird von ihm zur Betrachtung immer wieder zurückgerufen. Der Betrachter ist ein bisschen dumm; er ist nicht wie du und ich. Immer abrufbereit, eilt er vor jedes neue Werk, das seiner Gegenwart bedarf.“ (O'Doherty 1996: 39 f.)

Hatte O'Doherty noch an „den Betrachter“ gedacht, von dem wir in den Texten der Kunstkritik und -wissenschaft immer wieder lesen können (,Der Effekt auf den Betrachter ist [...]"), zeigt Ullrich in seinem Essay, wie sich an die soziale Position des Kunstbetrachters soziale Verhaltens- und Einstellungserwartungen angeheftet haben und ein „strenger Verhaltenskodex“ (Ullrich 2007:. 201 f.) ausgebildet wurde, der normiert, wie wir uns vor dem Kunstwerk zu präsentieren haben. Die Kunst selbst hat in vielfacher Weise auf diese Normierung reagiert, und es ist interessant zu sehen, wie die soziale Position des KUNSTSAMMLERS, auf die wir noch zurück kommen, wohl nicht zufällig mit einer ganz anderen räumlichen Positionierung neben oder sogar auf dem

5 Dirk vom Lehn und Christian Heath sprechen in vergleichbarem Zusammenhang von der „Konfiguration von Standpunkten“ (Vom Lehn/Heath 2007, S. 154; vgl. auch Heath/Vom Lehn 2004).

6 Begriffe für soziale Positionen werden im Beitrag durch Kapitälchen hervorgehoben. Dabei wurde versucht, an bereits bestehende und im Material selbst auftretende Begriffe anzuknüpfen. 
(verpackten!) Kunstwerk einhergeht (vgl. dazu die bei Ullrich (2007), S. $217 \mathrm{ff}$. wiedergegebenen Abbildungen von Kunstsammlern).

Die soziale Positionierung des KUnSTBETRACHTERS ist mit dem Gesagten allenfalls angedeutet, vielleicht aber genügend illustriert, um als solche in ihrer ganzen Unwahrscheinlichkeit deutlich hervorzutreten. Interessant im vorstehenden Zusammenhang ist, dass sie mit einer bestimmten Art der Lösung der kommunikativen Aufgabe des Bezugnehmens einher geht: nämlich dem ZurSchau-Stellen fokussierter Wahrnehmung, mit dem die Wahrnehmung des Kunstwerks selbst wahrnehmbar wird (Hausendorf 2003).

In der Schriftlichkeit ist dieser Weg in der Regel versperrt. Es ist, vereinfacht gesagt, nicht ohne weiteres möglich, auf das fragliche Kunstwerk zu zeigen, um das Bezugnehmen kommunikativ zu lösen. Wenn es also nicht über die wechselseitige Wahrnehmung unter Anwesenden geht, wie geht es dann? Wir wollen zur Illustrierung einen Blick in einen Ausstellungskatalog werfen und zwar auf den Beginn des Beitrags von Wilhelm Genazino im Katalog des Zürcher Kunsthauses zur Seurat-Ausstellung 2009/2010:

Der Beitrag beginnt auf einer rechten Seite. Wenn man den Band an dieser Stelle aufschlägt, fällt der Blick auf der linken Seite auf eine ganzseitige, farbige Reproduktion eines Bildes von Seurat. Links unten auf der rechten Seite (mit dem Text von Wilhelm Genazino), bündig abschließend mit der letzten Zeile des Textes, findet sich ein kleiner gedruckter Schriftblock, der durch seine Platzierung (linksbündig nach innen versetzt) vom Beginn des Textes abgegrenzt ist. Auf diesem kleinen Stückchen Text ist zu lesen:

Beispiel (2): eine Beschriftung

\author{
Georges Seurat \\ Une Baignade, Asnières \\ (Badeplatz bei Asnières), \\ Detail, 1883/84 \\ Öl auf Leinwand, $201 \times 300 \mathrm{~cm}$ \\ The National Gallery, London
}

Man kann vielleicht sagen, dass dieses kleine Stückchen Text (mit seiner soeben grob beschriebenen und wiedergegebenen druckschriftlichen Gestaltung) das Problem löst, von dem wir hier reden; die Angabe eines Personennamens, eines Titels (mit Übersetzung), eines spezifizierenden Hinweises auf die Art der Reproduktion, einer Jahreszahl, von Materialien und Format und eines Museums kann als stark musterhafte Lösung für das Problem der Bezugnahme auf das Kunstwerk gelten, die als kommunikative Gattung bzw. Textsorte in der Kunst- und speziell der Ausstellungskommunikation weit verbreitet ist. Beschriftungen dieser Art, zu deren Musterhaftigkeit gehört, dass sie irgendwie in der Nähe eines hervorgehobenen Objektes platziert sind, gehören zu jenem oben besprochenen „Zeigegestus“, mit dem ein Etwas nicht nur da, d. h. hier: sichtbar, ist, sondern in seinem „Da-Sein“ und seinem Anschauungswert demonstriert wird. Das einschlägige sprachliche Mittel dafür ist der Titel, mit dem das Werk zugleich identifiziert werden kann: Die Abbildung auf der lin- 
ken Seite zeigt ein Detail von „Badeplatz bei Asnières“. Wenn wir davor stünden, könnten wir auch sagen: das hier ist „Badeplatz bei Asnières“ von Seurat.

Die identifizierende Bezugnahme durch einen Eigennamen muss vom „wahrnehmungsabhängigen Bezugnehmen“ (von Polenz 1988: 120) unter Anwesenden, das wir zuvor beschrieben haben, unterschieden werden. Mit dem linguistisch gut beschreibbaren Unterschied der Referenz (vgl. dazu z. B. von Polenz 1988: 121 f.) geht zugleich ein Unterschied in der Pragmatik der Bezugnahme einher. Wenn wir ihn auf den uns interessierenden Aspekt der sozialen Positionierung beziehen, könnte man sagen: Im identifizierenden Bezugnehmen auf ein Werk macht sich ein Bedarf bemerkbar, der nicht auf die Unmittelbarkeit der Kunstbetrachtung zurück geht, sondern auf die Möglichkeit, eindeutige Referenz situationsunabhängig zu ermöglichen und zu suggerieren, dass es das wirklich gibt, worauf mit dem Namen hingewiesen wird. Und sagt uns der auf der Beschriftung getriebene Aufwand, der ja über den Titel deutlich hinausgeht (s. o. Bsp. 1), nicht auch genau das: Es gibt dieses Werk mit dem Namen „Badeplatz bei Asnières" tatsächlich, es ist in Wirklichkeit so und so groß, es wurde aus den und den Materialien geschaffen, und es befindet sich normalerweise da und da?

Wenn es so wäre, machte sich im identifizierenden Bezugnehmen eine soziale Positionierung bemerkbar, die primär mit dem Aufbewahren und der Erfassung, dem Sammeln und dem Zeigen (Ausstellen) und schließlich auch dem An- und Verkaufen von Kunstwerken, mit Logistik, Archivierung und Geldanlage zu tun hat. Es ist der Kunstbetrieb als Teil des Wirtschaftssystems, der sich an dieser Stelle innerhalb der Kunstkommunikation bemerkbar macht. Wir wollen die dazu gehörige soziale Position die Position des KunstsammLERS nennen. ${ }^{7}$ In ihr manifestiert sich die soziale Praxis des Ausstellens auf markante Weise. Ihr protoypischer sprachlicher Ausdruck ist die identifizierende Bezugnahme, wie sie z. B. in der Beschriftung eine musterhafte Ausprägung erfahren hat. Sie kommt auch dann zu ihrem Recht, wenn ein Werk, aus welchen Gründen auch immer, keinen Titel hat - und genau das zum Titel wird (,Untitled“). ${ }^{8}$ Kunstkommunikation, die über das anwesenheits- und momentgebundene Bezugnehmen auf das Werk, wie es durch und mit Wahrnehmungswahrnehmung entsteht, irgendwie hinaus gehen will, ist auf die Möglichkeit identifizierender Bezugnahmen wie selbstverständlich angewiesen. Man sollte nicht vergessen, dass sich darin immer auch und wie so oft auf sehr unscheinbare Weise die Integration der Kunstkommunikation in den Kunstbetrieb bemerkbar macht. Wann immer identifizierend von einem Werk die Rede ist, ist diese Rede neben allem anderen, was sie noch sein mag, auch ein Beitrag zur Konstitution des Warenwertes des Kunstwerks im Kunstbe-

7 Wolfgang Ullrich zeigt in dem bereits zitierten Beitrag (Ullrich 2007, S. 214 ff.), dass gegenwärtig die soziale Position des KunSTSAMMLERS einem Wandel zu unterliegen scheint, mit dem das Besitzen von Kunst viel wichtiger wird als das Betrachten und Zeigen von Kunst - und dass man darin durchaus eine Gegenreaktion auf den „etwas dummen“ KUNSTBETRACHTER sehen kann (s. o.). Vgl. dazu z. B. Vogt (2006). 
trieb. Anders als die Preisangabe, die bereits direkt auf Geld als Medium des Wirtschaftssystems verweist und von daher innerhalb der Kunstkommunikation wie ein Fremdkörper wirken mag, ist die identifizierende Bezugnahme auf das Werk noch gleichsam unschuldig. Sie dient nur, so die Botschaft, der notwendigen Identifizierung.

Wir wollen mit diesen Hinweisen auf zwei verschiedene Mittel des Bezugnehmens - das auf der Zur-Schau-Stellung fokussierter Wahrnehmung beruhende Zeigen und das auf der Benennung beruhende Identifizieren - und die ihnen nahe stehenden sozialen Positionen - die des KunstBETRACHTERS und die des KunstSAMMLERS - die Bemerkungen zur Aufgabe des Bezugnehmens vorerst abschließen ${ }^{9}$ und anhand der schon illustrierten Beschriftungen auf eine weitere Aufgabe der Kunstkommunikation, auf das Beschreiben, $\mathrm{zu}$ sprechen kommen.

\section{Beschreiben}

Wenn wir uns den Text der Beschriftung noch einmal in Erinnerung rufen, zeigt sich darin schnell ein kommunikativer Mehraufwand, der über das Bezugnehmen hinausgeht. Er zeigt sich z. B. - in dem durch den Normaldruck gegenüber dem Fettdruck in seiner Relevanz von vornherein herabgestuften Hinweis auf das verwendete Material und das Format des Werkes („Öl auf Leinwand, 201 x $300 \mathrm{~cm}$ “). Diese Hinweise unterstützen die identifizierende Bezugnahme auf das Werk dadurch, dass sie etwas thematisieren, das die Reproduktion innerhalb des Katalogs nicht mehr erkennen lässt: die Beschaffenheit und die Größe des Originals. Ich sehe darin eine für die Zwecke der Textsorte der Beschriftung stark formelhaft ausgeprägte Manifestation der Aufgabe des Beschreibens, die mit der Bezugnahme eng verbunden ist, aber darüber hinaus geht. Das Beschreiben soll hier als Antwort auf die Frage Was gibt es zu sehen (hören, tasten, schmecken, riechen)? verstanden werden. Es geht also um die Thematisierung dessen, was im Prinzip vor dem Kunstwerk der sinnlichen Wahrnehmung zugänglich ist. Das Beschreiben steht von daher speziell dem Zeigen als Mittel des Bezugnehmens nahe und tritt damit in vielen Fällen auch empirisch eng verbunden auf. Wenn man es so versteht, tritt die Besonderheit der hier zitierten beschreibenden Beschriftung („Öl auf Leinwand, 201 x $300 \mathrm{~cm}$ “) sofort hervor. Materialität und Format sind der sinnlichen Wahrnehmung zwar durchaus zugänglich (man kann im Prinzip sehen, könnte sicher auch fühlen, woraus ein Werk gemacht ist, und man kann sehen, könnte auch nachmessen, wie groß es ist), aber als Ausdruck einer Wahrnehmung vor dem Kunstwerk wäre eine Beschreibung wie „Ich sehe Öl auf Leinwand“ sofort

9 Wir werden später noch auf ein weiteres Mittel des Bezugnehmens zurück kommen, die klassifizierende Bezugnahme, die wir aufgrund ihrer Nähe zur sozialen Position des KUNSTKENNERs beim Erläutern besprechen. 
auffällig; ${ }^{10}$ sie würde uns in ihrer Musterhaftigkeit wie ein Zitat eines Beschriftungszettels oder -kärtchens vorkommen. Diese Musterhaftigkeit, die wir im Alltag wiedererkennen, ohne dass wir sie dafür analysieren müssen, hat sicher zu tun mit der Reduktion auf nicht weiter spezifizierte Stoffnamen, deren Verwendungszusammenhang elliptisch verkürzt durch ein PräpositionalAdjunkt ausgedrückt wird; all das konnotiert eine zurückgenommene Sachlichkeit, eine Zurückführung des Kunstwerks auf die Banalität der verwendeten Stoffe, die - ähnlich wie die Genauigkeit der Formatangaben - das gewissermaßen Handwerkliche des Werkes anklingen lässt. Wenn man sich fragt, worin die pragmatische Funktionalität einer solchen Beschreibung liegt, wird man im gegebenen Zusammenhang der Platzierung neben einer Reproduktion im Katalog an den oben schon genannten Verweis auf das „Original“ denken, also an das, was man gerade nicht sehen kann. In der Situation vor dem Original in einer Ausstellung, in der sich solche Beschriftungen typischerweise auch finden, können solche Hinweise, zumindest mit Bezug auf die Materialität des Werkes, tatsächlich auch Wahrnehmungshilfen liefern (,es ist wirklich Blut“, s. o. Anm. 10). Stärker ist m. E. aber der Hinweis auf den Akt der Erfassung der Daten des Werkes, wie wir ihn auch aus anderen Archiven kennen. Dazu passen die weiteren Angaben des Entstehungsjahres und des regulären Aufbewahrungsortes, die nichts mehr mit dem Beschreiben zu tun haben, sondern bereits zum Erläutern gehören (s. u. 5) und stärker noch als das Beschreiben auf das Sammeln und Bewahren verweisen. Das Beschreiben durch das Mittel der stark formelhaften Hinweise auf Material und Format ruft so gesehen die bereits besprochene soziale Positionierung des KUNSTSAMMLERS auf. So lässt sich übrigens auch gut erklären, woraus die stark kunstschaffende Kraft dieser Beschriftungstexte resultiert. Es ist das durch den Akt des Sammelns und Archivierens beglaubigte Attribut von Kunsthaftigkeit, das dem Werk durch diese Art des Beschreibens auf denkbar unscheinbare, fast subversive Weise verliehen wird.

Nicht alle Mittel des Beschreibens stehen in diesem pragmatischen $\mathrm{Zu}$ sammenhang. Wenn wir uns anderen Mitteln des Beschreibens zuwenden, wird die Beziehung zur sozialen Position des KUnSTBETRACHTERS evident. Wir wollen dazu einen Blick auf den Anfang des Textes von Wilhelm Genazino werfen:

\section{Beispiel (3): Sichtbarkeit}

„Auf vielen seiner Bilder zeigt uns Georges Seurat unverbunden beieinander sitzende, liegende oder stehende Menschengruppen, die gemeinsam in eine Richtung schauen. Als Bildbetrachter wissen wir nicht, ob sich die Leute kennen und ob sie bestimmte Interessen mit-

10 Was es empirisch gibt, sind Äußerungen wie ,weiß gar nich was für material das isch“ mit weiteren Thematisierungen möglicher Antworten (,öbis zwischen himbeermarmelade und bluet find ich“), die tatsächlich auf Möglichkeiten der sinnlichen Wahrnehmung vor dem Kunstwerk verweisen (,würds am liebste aalänge“) und insofern prototypisch für das Beschreiben sind (vgl. zum Kontext dieser Äußerungen und zur Wiedergabe des entsprechenden Gesprächsausschnittes Hausendorf 2007a: 28 f.). 
einander teilen oder nicht. Die Bildbetrachter sehen auch nicht, was die abgebildeten Figuren sehen. Das Interesse auslösende Ereignis befindet sich außerhalb der Bilder. Wir wissen nicht einmal, ob es ein solches Ereignis überhaupt gibt. [...]“ (S. 61)

Wenn man sich fragt, was die Frage ist, auf die diese ersten Zeilen eine Antwort sind, dann stößt man auf die für das Beschreiben konstitutive Frage (Was gibt es zu sehen?). Die Antwort im Text ist: ,unverbunden beieinander sitzende, liegende oder stehende Menschengruppen, die gemeinsam in eine Richtung schauen." Das Beschreiben wird hier nicht durch Hinweise auf Material und Format realisiert (wie auf der Beschriftung), sondern durch Hinweise auf Abgebildetes (,Menschengruppen“; „Leute“; „Figuren“). Einen leichten Überschuss an Sinn liefert uns der Text schon dadurch, dass er uns sagt, dass es „Georges Seurat“ ist, der „uns“ diese „Menschengruppen“ zu sehen gibt (,,zeigt"). Wir folgen also dem Künstleragens, wenn wir „unverbunden beieinander sitzende, liegende oder stehende Menschengruppen, die gemeinsam in eine Richtung schauen“ beschreiben. Was hier anklingt, ist schon mehr als nur ein Hinweis auf Abgebildetes. Es deutet sich ein Motiv an (eine Personengruppe als Motiv), das man mehr (wieder)erkennen als sehen kann. Darin steckt ein Überschuss in Richtung Erläutern (s. u. 4). In den folgenden Zeilen kehrt der Text dann aber wieder zum Beschreiben zurück, indem er die zuvor unauffällig indizierte Wir-Gruppe (,uns“) als Gruppe der „Bildbetrachter“ explizit einführt (,Als Bildbetrachter wissen wir nicht, [... ") und in ihrer primären Eigenschaft als Betrachtende und Sehende profiliert: „ob sich die Leute kennen [...] oder nicht“, können „wir“ nicht sehen; auch „,das, was die abgebildeten Figuren sehen“, „,sehen“"wir nicht. Und was wir als Betrachtende nicht sehen (können), können wir auch nicht „,wissen“. Diese Reflexionen, die das Sichtbare zur Sprache bringen, indem sie u. a. das Unsichtbare thematisieren, können als eine charakteristische Expandierung des Beschreibens gelten, die wir aus vielen anderen Zusammenhängen, in denen über Bilder geschrieben oder gesprochen wird, kennen (Hinweise auf Sichtbarkeit).

Die Betonung dessen, was es zu sehen gibt, ist gleichsam ein Appell an den KUNSTBETRACHTER, dessen vordringliche Aktivität die des Betrachtens und Sehens ist. Als solcher wird er angesprochen und in Anspruch genommen, nicht nur in der schriftlichen Kommunikation, sondern auch und gerade in der face-to-face-Situation vor dem Kunstwerk, in der der BILDBETRACHTER durch Kunstführer angeleitet wird, seiner sozialen Position entsprechend gerecht $\mathrm{zu}$ werden und vor allem zu ,sehen“:

Beispiel (4): ,sehen“

\begin{abstract}
„Da sehen Sie, sagte sie (eine Führerin im kunsthistorischen Museum; Hinzufügung von mir, hh), sehen Sie den Mund, da, sehen Sie, sagte Sie, diese weitausladenden Ohren, da, sehen Sie dieses zarte Rosa auf der Engelswange, da, sehen Sie im Hintergrund den Horizont, als ob nicht jeder auch ohne diese stupiden Bemerkungen alles das auf den Tintorettobildern gesehen hätte." (Thomas Bernhard, Alte Meister, S. 130).
\end{abstract}

Für den Ich-Erzähler in Thomas Bernhards Roman kommt in dieser Art von Beschreibung, die das Sichtbare explizit zur Sprache bringt, indem sie das Sehen der Besucher dirigiert, das eitle „Kunstgeschwätz“ der Museumsführer 
zum Ausdruck. Auch dabei erscheint der BILDBETRACHTER „ein bisschen dumm“ (s. o. 2): „Die Führer in den Museen behandeln die ihnen Anvertrauten doch immer nur als Dummköpfe, immer als die größten Dummköpfe" heißt es wenig später - recht passend - bei Thomas Bernhard im Anschluss an die gerade zitierte Stelle.

Der Beginn des Textes von Wilhelm Genazino beschränkt sich keineswegs auf Hinweise auf Sichtbarkeit. Wir hatten darauf oben bereits mit dem Übergang vom Sichtbaren zum Motiv aufmerksam gemacht. Wohl nicht zufällig taucht wenig später im Anschluss an die zitierte Eingangsstelle der Begriff „Motiv“ im Text selbst auf. Der Autor thematisiert damit das Nicht-Sichtbare, wiederum aus der Perspektive des „Bildbetrachters“:

Beispiel (5): „Motiv““

„Das (= Industrialisierung, Ödnis der Städte, Enge der Nachbarschaft, Eingezwängt-Sein in den Wohnungen, [...]; Hinzufügung von mir, hh) sind Motive, die dem Bildbetrachter zwanglos-zwanghaft einfallen müssen, gerade weil sie nicht abgebildet sind." (S. 62)

Darin zeigt sich sehr unmittelbar, dass der Hinweis auf Motive auch dann greift, wenn letztere nicht sichtbar sind, sondern dem „Bildbetrachter zwanglos-zwanghaft einfallen müssen“. Aus dem Beschreiben wird mit diesen Hinweisen auf Motive ein Erläutern - es geht um etwas, das man wissen kann oder muss (s. u. 4) -, das der Autor an dieser Stelle aber dem „Bildbetrachter“ und sich selbst (in der Position des KunstBETRACHTERS) gleichsam unterschiebt, ohne sich in die Position des KunSTKENNERS (der erläutert, was es zu sehen gibt) zu begeben. So kann der Autor dann im unmittelbaren Anschluss seiner eigenen Verwunderung darüber Ausdruck verleihen, gleichsam wie von selbst als KUNSTBETRACHTER zum KUNSTKENNER geworden zu sein:

\section{Beispiel (6): vom KUNSTBETRACHTER zum KUNSTKENNER}

„Wir sind jetzt überraschend schnell in das mögliche innere Zentrum der Bilder vorgestossen: Das Nicht-Abgebildete war das vorerst nicht adäquat Darstellbare. Dazu bedurfte es der fortgeschrittenen Moderne, in der wir uns (auf diesen Bildern) noch nicht befinden." (S. 62)

Mit diesem Hinweis auf die „fortgeschrittene Moderne“ im Sinne einer bestimmten Kunstepoche bzw. eines bestimmten Stils positioniert sich der Autor ohne Zweifel als KUNSTKENNER, aber als einer, der gleichsam als (unbedarfter) KunSTBETRACHTER daherkommt. Darin liegt, wenn man so will, der Kunstgriff des Textes von Genazino.

\section{Erläutern}

Das Erläutern, von dem wir bereits gerade gesprochen haben, wollen wir so verstehen, dass es über das hinaus geht, was prinzipiell aus der sinnlichen Wahrnehmung des Kunstwerks erschließbar ist und deshalb gewusst werden muss bzw. gewusst werden kann. Das Erläutern antwortet also auf die Frage, was man über das Kunstwerk weiß. So wenig, wie es für das Beschreiben notwendig ist, dass es explizit eingeleitet werden muss mit einem verbum 
sentiendi (,ich sehe auf dem Bild [... “), ist es für das Erläutern notwendig, dass es mit einem Verb, das auf Wissen und Vertrautheit verweist, eingeleitet werden muss (,,ich weiß über das Bild, dass [... $]^{\circ)}$ ). Wenn es bei Wilhelm Genazino z. B. heißt, dass es der „fortgeschrittenen Moderne bedurfte“, um das bei Seurat „Nichtabgebildete“ adäquat darzustellen (s. o. Bsp. 4), dann löst sich der Autor von dem, was man auf den Bildern sehen und beschreiben kann zugunsten dessen, was man über das Bild als Werk einer bestimmten Epoche und über die Kunstgeschichte wissen kann. Es steckt also die Suggestion eines Wissens (über die „fortgeschrittene Moderne“) hinter dieser Aussage, die dazu dient, die zuvor aufgestellte Behauptung (zum ,Zentrum der Bilder vorgestoßen“" zu sein) zu erläutern. In ähnlichem Kontext finden wir eine weitere Erläuterung:

\section{Beispiel (7): Hinweis auf „Technik“}

„Tatsächlich sind die Themen-Bilder genauso starr und reglos wie die Menschen-Bilder. Dazu passt, dass auf den Themen-Bildern keine Menschen (oder nur sehr wenige) zu sehen sind und dass diese wenigen genauso statisch erscheinen wie die Objekte ringsum. Dieser Eindruck geht zurück auf die von Seurat entwickelte Punkte-Technik. Es könnte sein, dass erst diese Punkte-Technik aus Seurat einen Neo-Impressionisten gemacht hat - und zwar insofern, weil nur durch die Punkte-Technik eine organische Abwendung vom konventionellen Impressionismus der (sagen wir mal so:) Technik der schaukelnden Striche möglich war." (S. 64)

Zunächst wird eine Beschreibung geliefert, die einer geben kann, der sich die Bilder genauer angeschaut hat und dem aufgefallen ist, was (nicht) ,zu sehen“ ist und welchen „Eindruck“ das auf den KUNSTBETRACHTER haben mag. Der Hinweis auf die „Punkte-Technik“ dient dann dazu, diesen „Eindruck“ zu erläutern mit Hilfe der Malweise, von der weiter gesagt wird, dass ,erst“ sie ,aus Seurat einen Neo-Impressionisten gemacht hat“. Zu dem Hinweis auf die Technik kommt also zusätzlich noch ein erläuternder Hinweis auf Stil und Epoche. Das schreibt einer, der suggeriert zu wissen, was „die von Seurat entwickelte Punkte-Technik“ ist und was einen „Neo-Impressionisten“ von den Impressionisten unterscheidet. Mit diesen Erläuterungen geht also eine Darstellung von Kennerschaft und Expertise einher, mit der sich der Verfasser als KUNSTKENNER positioniert; als einer, der „die Geschichte der Malerei gut kennt und über den Platz, den Seurat darin einnimmt, zu reden weiß. " ${ }^{11} \mathrm{Zu}$ der sozialen Position des KUNSTKENNERS gehört insofern eine gewisse Beredsamkeit, in der sich das fragliche Wissen wortreich zum Ausdruck bringen lässt. Wilhelm Genazino geht in seinem Text mit dieser dem KUNSTKENNER eigenen Beredsamkeit sehr zurückhaltend um: Im oben wiedergegebenen Ausschnitt (Beispiel 7) z. B. wird mit dem umschreibenden Kompositum „PunkteTechnik“ gezielt ein Fachwort wie „Pointillismus“ vermieden; der Ausdruck „Technik der schaukelnden Striche“, der wenig später kontrastierend gebraucht

11 Barthes (1983), S. 89; im Original steht statt „Seurat“ „Twombly“, weil es im Text von R. B. um den Maler Cy Twombly geht. Barthes umschreibt diese Position als „Subjekt der Spezialisierung“ (ebd.); s. auch oben das Zitat in Kap. 2. 
wird, wird mit einer Formel eingeleitet, die explizit auf den Ad-hoc-Charakter der Umschreibung verweist (,sagen wir mal so“). Die These von dem, was Seurat zum Neo-Impressionisten gemacht hat, wird mit einer starken Modalisierung („Es könnte sein“) in ihrem Geltungsanspruch von vornherein stark eingeschränkt. Zumindest stilistisch wird auf diese Weise die soziale Positionierung im Sinne der Darstellung von Expertise und Kennschaft abgeschwächt. Und auch an anderen Stellen in Wilhelm Genazinos Text findet man solche Abschwächungen des Erläuterns - etwa wenn Hinweise auf den (sozial)historischen Hintergrund der Entstehung der Bilder gegeben werden:

\section{Beispiel (8): Hinweise auf den historischen Hintergrund}

„Ein interesseloses Wohlgefallen am bloßen In-der-Zeit-Sein können wir heute kaum noch nachvollziehen. Insofern neigen wir Heutigen dazu, ihnen geläufige Inhalte zu unterlegen.

Es bietet sich zum Beispiel an von der eben aufsteigenden Fremdheit zwischen den Menschen zu sprechen. Tatsächlich eignen sich Seurats Bilder für diese These ganz ausgezeichnet. Man könnte behaupten: Mit Seurat dringt die Fremdheit in den Impressionismus ein - die Distanz, die Differenz, das Erschrecken. Ich könnte weiter behaupten: Die damals neu aufsteigende Fremdheit zieht eine künstliche Selbstversunkenheit der Subjekte nach sich.“ (S. 62)

Einerseits wird deutlich, dass auch hier einer spricht, der ein Wissen über den relevanten historischen Hintergrund hat und die gesellschaftliche Situation „heute“ von der „damals“ unterscheiden kann; einer, der weiß, dass es die „Fremdheit zwischen den Menschen“ ist, die in der Zeit des ausgehenden 19. Jahrhunderts dabei ist ,aufzusteigen“, und der dieses Wissen erläuternd einsetzt, um über die Bilder zu sprechen. R. Barthes hat diesen Sprecher das „Subjekt der Kultur, der Bildung“ genannt (s. o. 2). Andererseits wird genau die hier im Text aufsteigende Beredsamkeit selbst distanzierend zur Sprache gebracht: durch die selbst- und rückbezügliche Thematisierung des Thematisierens der „Fremdheit“ (,Es bietet sich zum Beispiel an, von [...] zu sprechen“; „,diese These“; „Man könnte behaupten“; „Ich könnte weiter behaupten:“), durch die erneute Einschränkung des Geltungsanspruchs (,könnte behaupten") und durch die Wiederholung genau dieser Distanzierungsformen (,könnte weiter behaupten“). Mit all dem strömt das fragliche Wissen nicht ungebremst in die Kommunikation, sondern gleichsam durch den Filter einer leichten, aber nicht überlesbaren Distanz.

Man erkennt diese Distanzierung besonders deutlich, wenn man Wilhelm Genazinos Text mit dem ihm unmittelbar folgenden Text im Ausstellungskatalog vergleicht. Ich zitiere aus den einleitenden Sätzen:

\section{Beispiel (9): KUNSTKENNER}

„Das Werk Georges Seurats (1859-1891) erscheint heute als ein Stern erster Größe in der
weitläufigen historischen Konstellation der Moderne. [...] Seurats historische Größe, diese
Behauptung darf man wagen, hat damit zu tun, dass er mit Malerei und Zeichnung einen neu-
en Anfang gemacht hat. Wir werden genauer sehen, worin er besteht. Jedenfalls kam er nicht
unvermittelt zustande, sondern schloss eine Fülle von Auseinandersetzungen ein: mit der
Tradition der Kunst, einschließlich der Alten Meister, mit den rezenten Bildvorstellungen der 
Impressionisten, mit der sich dramatisch verändernden sozialen und urbanen Umwelt der Metropole Paris, mit der neuen und dominanten Leitwissenschaft der zweiten Hälfte des 19. Jahrhunderts: einer physiologischen Psychologie, die sich nicht zuletzt mit Gesetzen und Wirkungsweisen der Farbe beschäftigt hatte und mit dem insbesondere literarischen Symbolismus, der von Richard Wagner angeregt war und zu dem Seurat in seinen letzten Lebensjahren hintendierte [...].“(S. 85 f.)

Man muss nicht wissen, dass dieser Text von Gottfried Boehm stammt ${ }^{12}$, um darin die charakteristische Beredsamkeit des KUNSTKENNERS wiederzuerkennen. Das fängt bereits mit den ersten Worten an, einer klassifizierenden Bezugnahme auf die fraglichen Bilder mit dem Hinweis auf das „Werk“ des Künstlers („Das Werk Georges Seurats“), geht über die Auflistung der für die „umfangreiche wissenschaftliche Literatur zu Seurat“ (im Anschluss, in Beispiel 7 nicht mehr wiedergegeben) maßgeblichen „Stichworte“ und endet im Zitat mit einem Hinweis auf die für die späteren Arbeiten wichtigen biographischen Einflüsse (,in seinen letzten Lebensjahren“). Es ist ein Kunstwissenschaftler, der hier spricht und der von Beginn an keinen Hehl daraus macht, dass es im Folgenden auch und gerade um Wissenskommunikation von und für Experten geht. Manifester Ausdruck dieser Orientierung: die verwendete Fachsprachlichkeit.

Neben dem Erläutern, das mit seiner Orientierung an dem, was man wissen kann und vielleicht auch wissen sollte, ist zudem das Bewerten in dem gerade wiedergegebenen Beleg sehr präsent: Der Text beginnt damit (,ein Stern erster Größe“), und die in Aussicht gestellten Erläuterungen (,Wir werden genauer sehen") verstehen sich als Begründung für die vorangestellte Wertung (,Seurats historische Größe“). Wir werden darauf noch zurück kommen, wollen aber zuvor noch auf das Deuten zu sprechen kommen.

\section{Deuten}

Es gibt eine Alternative zum Erläutern, die ebenso über das Beschreiben des Sichtbaren hinausgeht und auf etwas abzielt, was gewissermaßen hinter dem Sichtbaren steht und dem Sichtbaren einen speziellen Sinn verleiht: das Deuten. Es zielt auf das Verstehen des Kunstwerkes. Anders als das Erläutern, das Wissen suggeriert, erfolgt das Deuten typischerweise im Modus des „unsicheren Für-Wahr-Haltens“ (von Polenz 1988). Es geht beim Deuten also nicht um etwas, das epistemisch verbürgt und begründet werden kann, sondern um etwas, das gewissermaßen in den Raum gestellt wird im Sinne einer Interpretation, die um ihren interpretativen Charakter weiß. Im Text von Wilhelm Genazino gibt es dafür einen anschaulichen Beleg, in dem der Verfasser auf mögliche Intentionen des Künstlers hinweist:

12 „Gottfried Boehm“, so die Kategorisierung im Vorwort des Katalogs, „der Kunsthistoriker und Philosoph, der über die Kunst- und Wahrnehmungstheorie der Renaissance, des 19. und 20. Jahrhunderts geschrieben hat, ist seit 1986 Ordinarius für Kunstgeschichte [...]“. 


\title{
Beispiel (10): Hinweis auf Künstlerintentionen
}

\begin{abstract}
„Auf dem einen oder anderen Bild hat uns Seurat erlaubt, die Magie als Bildmoment zu erkennen. Zu diesen Bildern zähle ich (zum Beispiel) die Kreidezeichnung Au concert Européen [...] oder das Porträt Jeune Femme se poudrant [...], in der wir Seurats Freundin Madeleine Knoblock erkennen dürfen. Auf dem Konzertbild ist nicht die Sängerin auf der Bühne das Hauptthema, sondern die merkwürdigen Frisuren der Frauen im Vordergrund. Ist es erlaubt, in den hoch aufgerichteten Haar-Türmen der Frauen eine versteckte und dann doch eingestandene Belustigung des Malers zu sehen? Und ist es erlaubt, im Porträt der Freundin die sanfte Komik des (sagen wir:) ,hochgerüsteten Körpercharmes der Damenwelt zu erblicken"? Natürlich kann ich die hier angesprochenen Bildmomente nur in Frageform vorbringen; es ist möglich, dass Seurat gleichzeitig ein ernster Mensch war und dass er die belustigende Distanz, die ich ihm hier unterstelle, zurückgewiesen hätte. Aber daran glaube ich nicht; die auf den Bildern mit gemalte Magie ist ein Freiraum, den Seurat gezielt absichtlich genutzt hat.“ (S. 66)
\end{abstract}

Die Deutung wird gleich zu Beginn des Zitats mit einer Formulierung vorbereitet, die auf „Seurat" als Künstleragens hinweist, der in seinen Ausdrucksintentionen für uns ,„auf dem einen oder anderen Bild“ transparent(er) wird. Die Bezugnahme auf die fraglichen Bilder lässt mit der klassifizierenden Referenz (,die Kreidezeichnung“; „,das Porträt“) und der Darstellung biographischen Hintergrundwissens (,Seurats Freundin Madeleine Knoblock“) zunächst die soziale Position des KUNSTKENNERS anklingen - die dann aber mit der vorangestellten Infragestellung des Geltungsanspruches („Ist es erlaubt“) der nachfolgenden Deutung sofort wieder zurückgenommen wird. Verstärkt wird diese explizite Markierung „unsicheren Für-Wahr-Haltens“ durch die gleich anschließende Wiederholung (,Und ist es erlaubt“) und die nachträgliche metakommunikative Betonung der Fraglichkeit der vorgetragenen Deutungen (,[...] nur in Frageform vorbringen“). Der, der so spricht, spricht nicht mit dem Wissen des KUNSTKENNERS, sondern mit der Unbefangenheit des KUNSTBETRACHTERS, der gewissermaßen ungeschützt und unbedarft (,Ist es erlaubt“) in dem, was er sieht (,hoch aufgerichtete Haar-Türme“; „Porträt der Freundin"), etwas anderes erkennt, was im Sichtbaren bloß ausgedrückt, aber mit verstanden werden darf (oder eben nicht mit verstanden werden darf): eine „Belustigung des Malers“ über „,die sanfte Komik des [...] hochgerüsteten Körpercharmes der Damenwelt“". Zum Deuten gehört, wie eingangs erwähnt, dass es nicht mit Wissen abgesichert ist - was die dem Künstler gleichsam unterschobenen (ihm ,unterstellten"), von ihm aber gerade nicht mitgeteilten Intentionen zu einem bevorzugten Mittel des Deutens macht. In ihrem Nachvollzug erst, so die Botschaft, kommt das Verstehen der Bilder zum Abschluss (,die auf den Bildern gemalte Magie ist ein Freiraum, den Seurat gezielt absichtlich genutzt hat"). Dazu ist es auf das Deuten, in all seiner Unsicherheit, angewiesen.

Wie soeben angeklungen ist, neigen wir dazu, das Deuten (z. B. in Form des hier illustrierten Hinweises auf eine versteckte Intention des Künstlers) als eine mit der sozialen Position des KunSTBETRACHTERS verbundene Praxis zu verstehen. Dem Künstler selbst muss das Deuten aus naheliegenden Gründen Unbehagen verursachen. Der KUNSTKENNER wird sich dagegen wehren, weil er ansonsten Wissen durch Spekulation ersetzen müsste. Aber der KunSTBET- 
RACHTER ist darauf angewiesen, weil er in seiner Position vor dem Kunstwerk immer schon in einen kommunikativen Zusammenhang geraten ist, mit dem er irgendwie verstehend fertig werden muss. Auch im Deutungskontext steht der KUNSTBETRACHTER also wieder eigentümlich „dumm“ da (s. o. 3).

\section{Bewerten}

Wir können für das Bewerten zunächst auf unser an den Anfang gestelltes Beispiel aus der „Castelli-Galerie“ zurück kommen, um die Charakteristik des Bewertens als Antwort auf die Frage, was davon zu halten ist, zu verstehen und verschiedene Mittel des Bewertens zu illustrieren: die Darstellung

- der Wirkung bzw. des Eindrucks des Werkes auf den Betrachter (,,unglaublich. absolut unglaublich"; ,,ungeheuer"),

- des eigenen Gefallens des Werkes (,die gefallen Ihnen also?“; „selbstverständlich gefielen sie mir nicht so wie die Plexiglas-Skulpturen"),

- der Qualität des Werkes (,klasse, absolute Klasse“; , grandios. einfach grandios") und

- des Ranges und Wertes des Werkes (,ein Plagiat - ein armseliger Abklatsch von Diane Arbus, aber ohne ihren Witz").

Insbesondere in der Darstellung des künstlerischen Ranges des Werkes macht sich die soziale Position des KunSTRICHTERS bemerkbar, der den Wert des Werkes vergleichend beurteilt und gewissermaßen deklariert. ${ }^{13}$ Während die einen (Tracy und Isaac) ihre Subjektivität (Gefallen) und Affektivität (Eindruck und Wirkung) aus der Position des „etwas dummen“ KUNSTBETRACHTERS darstellen (Gefallen und Wirkung), deklariert die andere (Mary) den künstlerischen Rang des Wertes aus der Position der (selbsternannten) Autorität des KUNSTRICHTERS (die noch in der Szene selbst mit der Stilisierung ebenso schillernder wie nichtssagender Urteile karikiert wird: ,unwahrscheinlich strukturell $[\ldots]$ vollkommen integriert $[\ldots]$ eine phantastisch negative Potenz"). Speziell im Vergleich mit einer anerkannten Künstlerin, mit dem das vernichtende Urteil ausgesprochen wird (,ein armseliger Abklatsch von Diane Arbus"), macht sich die Expertise des KUNSTRICHTERS bemerkbar, aber sie ist hier - anders als beim KUNSTKENNER - ganz dem Bewerten untergeordnet.

Die Differenz dieser Positionen zeigt sich, wenn wir noch einmal auf den Beginn des Textes von Gottfried Boehm zurückkommen (s. o. Beispiel 9). Auch hier ist das Bewerten als Urteil über den künstlerischen Rang und Wert des Werkes im Text unmittelbar gegenwärtig:

Beispiel (11): Detail

„Das Werk Georges Seurats (1859-1891) erscheint heute als ein Stern erster Größe in der weitläufigen historischen Konstellation der Moderne. Längst zögert man nicht mehr, ihn zu den „Klassikern“ zu rechnen, [...]. Seurats historische Größe, diese Behauptung darf man

13 Die Deklaration des Ranges des Kunstwerkes hebt Christiane Thim-Mabrey als dominante Texthandlung der Konzertkritik hervor (Thim-Mabrey 2007: 106 f.). 
wagen, hat damit zu tun, dass er mit Malerei und Zeichnung einen neuen Anfang gemacht hat." (S. 85)

Aber es handelt sich weniger um eine Deklaration, mit welcher der Wert des Werkes erstmals festgestellt wird, sondern eher um das Konstatieren und das Zitieren einer bekannten Einschätzung (,erscheint heute als ein Stern erster Größe“; „Längst [...] zu den ,Klassikern“ zu rechnen“; „Seurats historische Größe"). Und anders als im ersten Beispiel dient hier das Bewerten vor allem dazu, unmittelbar zum Erläutern überzugehen: Es soll gezeigt werden („Wir werden genauer sehen“, s. o. Beispiel 7), worauf ein solches Werturteil beruht (,hat damit zu tun, dass er einen neuen Anfang gemacht hat“). Darin manifestiert sich die soziale Position des KUnSTKENNERS, der sich auf eine epistemisch abgesicherte Autorität - und nicht auf den Jargon der Kunstkritik beruft.

Viel spricht dafür, dass das Bewerten in der Praxis der Kunstkommunikation einen besonderen Stellenwert hat (s. o. 1). Dem entspricht die Vielfalt der Mittel des Bewertens, die sich keineswegs nur innerhalb der Position des KunSTRICHTERS finden. So wird der Wert des Werkes dem KUNSTBETRACHTER gewissermaßen schon durch das Ausgestellt-Sein der Werke, durch ihre Sammlung, Archivierung und Ausstellung suggeriert. Dass es sich lohnt, die Werke in Augenschein zu nehmen, ist gewissermaßen die stille Implikation jeder Ausstellung (die mit dem Zahlen des Eintrittspreises auch tatsächlich sinnfällig wird). Sie ist auch eine Zumutung für den KUNSTBETRACHTER, sich zu dieser Unterstellung zu verhalten: typischerweise mit der Darstellung von Subjektivität und Affektivität (s. o.). Auch das zeigt der vorangestellte Ausschnitt aus der „Castelli-Galerie“ mit großer Anschaulichkeit. Das Wahrnehmen und das Verstehen haben ihren Fluchtpunkt im Auf-Sich-Wirken-Lassen der Werke. Der KUNSTBETRACHTER ähnelt insofern immer auch jenem, dem ein Menu in einem Feinschmeckerrestaurant vorgesetzt wird, und der vor allem bekunden muss, ob es geschmeckt hat. ${ }^{14}$

\section{Fazit und Ausblick}

Im vorliegenden Beitrag werden an ausgewählten Beispielen typische Problemstellungen der Kunstkommunikation und die sprachlich-kommunikativen Antworten auf diese Probleme illustriert: das Bezugnehmen auf das Kunstwerk, das Beschreiben, das Deuten, das Erläutern und das Bewerten des Werkes. Dabei zeigt sich schnell, dass sich in der Art der Bearbeitung dieser Problemstellungen (z. B. in der Art und Weise des Bezugnehmens auf das Kunstwerk) nicht nur individuelle Sprechweisen, sondern charakteristische Aufgabenverteilungen und kategoriengebundene Sprechweisen des Kunstbetriebs manifestieren, dass sich, anders gesagt, im Bezugnehmen, im Beschreiben, im Deuten, im Erläutern und im Bewerten soziale Positionen manifestieren, wel-

14 R. Barthes spricht in diesem Zusammenhang vom „Subjekt des Vergnügens“ (s.o. 2), das „sprachfaul“ ist und im Grunde im Genuss des Werkes aufgeht. 
che die Kunstkommunikation im Kunstbetrieb gesellschaftlich verankern. Schon das vielleicht unscheinbarste (und deshalb oft übersehene) Mittel des Bezugnehmens, die Zur-Schau-Stellung fokussierter Wahrnehmung (s. o. 3), versteht sich nicht von selbst, sondern ist Ausdruck einer bestimmten sozialen Wirklichkeit des Kunstbetriebs, zu der es dazu gehört, dass ein als Kunstwerk erlebtes Objekt gleichsam mit Wahrnehmungsmuße behandelt wird, d. h. im Modus zweckfreier sinnlicher Wahrnehmung (Oevermann 1996) betrachtet wird. Auf diese gesellschaftlich (vor)geprägte soziale Wirklichkeit, die sich noch vor dem Sprechen und Reden über Kunst schon in der Annäherung an Kunst bemerkbar macht, soll die Rede von sozialen Positionen aufmerksam machen: Das Bezugnehmen auf das Kunstwerk in Form der Zur-SchauStellung fokussierter Wahrnehmung gehört zur sozialen Positionierung als Kunstbetrachter. Die Praxis der Ausstellungskommunikation im „White Cube" mutet mit dieser Form der Bezugnahme auf das Werk wie selbstverständlich die soziale Positionierung als KUNSTBETRACHTER zu - mitsamt ihren Folgelasten. Ob er oder sie es will oder nicht, vor dem Kunstwerk wird der Ausstellungsbesucher zum KUNSTBETRACHTER - und d. h.: mit den kommunikativen Erwartungen konfrontiert, die mit dieser Position verbunden sind (s. o. 3). Das Gleiche gilt übrigens, wenn wir einen Katalog (z. B. den, aus dem wir in diesem Beitrag vor allem zitiert haben) aufschlagen und dabei seitenweise auf aufwendig und ganzseitig abgebildete Reproduktionen (,Tafeln“) von Kunstwerken stoßen: Sie machen den Leser zum KUNSTBETRACHTER, ob er will oder nicht.

Welche Erwartungen mit dieser sozialen Position verbunden sind und ob und wie sie dann mit und durch das Beschreiben, das Deuten, das Erläutern und das Bewerten des Betrachteten kommunikativ eingelöst werden, ist eine empirische Frage. Man kann sie historisch beantworten und nach den Ursprüngen der Ausbildung einer sozialen Position des KunstBETRACHTERS fragen (vgl. Ullrich 2003), man kann danach fragen, was für ein Bild des KUNSTBETRACHTERS die Texte und die Ausstellungen des Kunstbetriebs implizieren (vgl. O’Doherty 1996), und man kann anhand der Dokumentation des Verhaltens und des Kommunizierens vor dem Kunstwerk in zeitgenössischen Kunstausstellungen auch gesprächslinguistisch und konversationsanalytisch untersuchen, welche Aktivitäten typischerweise mit der Einnahme der sozialen Position des KUNSTBETRACHTERS einhergehen. ${ }^{15}$ Was sich dabei abzeichnet, ist, dass es mit dem Bezugnehmen durch Wahrnehmungsmuße nicht getan ist: Es gibt Hinweise auf Abgebildetes und Sichtbares, um das Betrachtete zu beschreiben, Hinweise auf ein Künstleragens, welche das Betrachtete verständlich(er) machen und vor allem die Zumutung, sich dazu zu verhalten, was das Betrachtete mit einem selbst „macht“ (Bewerten: Hinweise auf Wirkung und

15 Man kann auch künstlerisch darauf reagieren - wenn man z. B. an die Museums- und Besucherfotographien von Thomas Struth denkt, auf denen wir Menschen dabei betrachten können, wie sie genau das tun, was wir auch gerade tun, wenn wir in einer Ausstellung eben diese Fotographien betrachten. Vgl. auch die Beispiele, die bei Ullrich (2007) vorgestellt werden. 
Eindruck, Gefallen und Missfallen). Wir haben das in diesem Beitrag vor allem mit einem Text von Wilhelm Genazino zu zeigen versucht, der nicht nur, aber sehr stark aus einer Position des KUNSTBETRACHTERS heraus geschrieben ist. Man könnte dazu aber auch auf Evidenz aus anderen empirischen Untersuchungen zur Position des KunSTBETRACHTERS verweisen (vgl. z. B. Hausendorf 2005; Hausendorf 2006; Vom Lehn/Heath 2007).

Bei einer solchen Analyse zeigt sich schnell, dass der KUNSTBETRACHTER nicht alleine vor dem Kunstwerk steht. Schon, dass er dort stehen kann, verdankt er in der Regel einem voraus laufenden Prozess des Sammelns, Aufhebens, Verwahrens, Ausleihens oder Kaufens und Ausstellens von Kunstwerken. Man stößt darauf, wenn man z. B. die Kärtchen studiert, die unweit vom Werk angebracht sind. Wir haben zu zeigen versucht, dass sich hier eine andere soziale Position bemerkbar macht, die vielleicht die des KUNSTSAMMLERS ist. Ihre typischen sprachlichen Mittel und Formen liegen, so die hier vorgestellte These, im identifizierenden Bezugnehmen und im Beschreiben durch den formelhaft ausgeprägten Hinweis auf Format und Material (s. o. 3). Man könnte behaupten, dass der KUNSTBETRACHTER und der KUNSTSAMMLER vor dem Kunstwerk miteinander in Kontakt treten: augenfällig dann, wenn z. B. Besucher regelmäßig zwischen der Betrachtung eines Werkes und der Lektüre der Kärtchen hin- und herpendeln. Im vorliegenden Beitrag kann lediglich angedeutet werden, dass und wie soziale Positionen im Sprechen und Schreiben über Kunstwerke zur Geltung kommen. Eine systematische Rekonstruktion dieser Positionen stellt ein echtes Desiderat dar. Das gilt nicht nur für den KUNSTBETRACHTER und den KUNSTSAMMLER, sondern auch für die sozialen Positionen des KunSTKENNERS und des Kunstrichters. Wir haben sie hier aufgeführt, weil sie in den Beispieltexten und -szenen, die behandelt wurden, bereits anklingen und als soziale Positionen im Kunstbetrieb fest etabliert sind: So gibt es eigene Textsorten - wie die Kunstkritik -, in denen die der jeweiligen sozialen Position zugrundeliegende Aufgabenstellung - wie das Bewerten - eine musterhaft ausgeprägte Gestalt bekommen hat (vgl. Thim-Mabrey 2007). Auch wenn man - wie im vorliegenden Beitrag - nur exemplarisch vorgeht, zeigen sich auch mit Blick auf diese sozialen Positionen typische Sprechweisen: Für die soziale Position des KUNSTRICHTERS ist offenkundig das Bewerten und hier speziell die Deklaration des Rangs des Werkes konstitutiv: Wer einem Werk den Rang abspricht (indem er es wie Mary zu einem „faden Abklatsch“ macht), positioniert sich damit unweigerlich als KUNSTRICHTER, der sich gleichsam über die Subjektivität des Geschmacksurteils mit der Autorität des Kritikers erhebt. Für die soziale Position des KUNSTKENNERS konnten wir eine Reihe von Mitteln des Erläuterns belegen, die auf unterschiedliche Weise Wissen über Kunst suggerieren, das der KUNSTBETRACHTER allein vermöge seiner Wahrnehmungssituation vor dem Werk nicht hat. Dazu gehören etwa der Hinweis auf Motive oder der Hinweis auf Kunstepochen.

Wenn man diese Beobachtungen etwas schematisch zusammen fassen wollte, ergeben sich eine Reihe von Relationen zwischen kommunikativen 
Aufgaben und sozialen Positionierungen: ${ }^{16}$ Auffallen mag, dass die soziale Position des KÜNSTLERS in dieser Übersicht nicht auftaucht. Manches spricht dafür, dass der KÜNSTLER in der Kunstkommunikation eben im Werk selbst präsent ist. Wenn er oder sie davor steht (und sei es vor dem eigenen Werk), wird er zwangsläufig zum KUNSTBETRACHTER - mit allen Implikationen und Ausweichmöglichkeiten (auch auf andere soziale Positionen, die dann noch bleiben). Man kann diese Überlegung z. B. dadurch fruchtbar machen, dass man fragt, ob und auf welche Weise die genannten sozialen Positionen nicht immer schon im Werk selbst präsent sind - als Vorwegnahmen und Thematisierungen der mit diesen Positionen verbundenen Erwartungen. Aber das ist eine Perspektive, die wir hier nicht weiter verfolgt haben. ${ }^{17}$

Die Übersicht lässt auch so schon keinen Zweifel daran, dass es sich bei den hier vorgestellten Überlegungen lediglich um eine Forschungsperspektive handelt, die erst noch empirisch und wohl auch methodologisch einzulösen wäre. Wenn sich soziale Positionen, wie behauptet, erst im Sprechen selbst konstituieren, darf man sie z. B. nicht mit vorgegebenen Attributen von Sprecher und Sprecherinnen verwechseln (wie sie z. B. mit Berufszugehörigkeit zu tun haben). Auch ein Kunstkritiker der Neuen Zürcher Zeitung kann sich vor dem Kunstwerk als ein KUNSTBETRACHTER positionieren. Und ein Mensch aus Fleisch und Blut ist nicht daran gehalten, sich vor dem Kunstwerk nur auf eine Weise zu positionieren. Wie immer man es auch drehen und wenden mag, soziale Positionierungen erweisen ihre Evidenz ausschließlich im Kommunikationsprozess selbst, z. B. darin, dass und wie sie auf eine bestimmte Weise hergestellt, aufrechterhalten und von Fall zu Fall auch wieder aufgelöst werden; dass und wie sie auf eine bestimmte Weise erwartbar gemacht werden und dass und wie in und mit Kommunikation darauf reagiert werden kann; bspw. darin, dass und wie sie unter Anwesenden eine spezifische Aufgabenverteilung nahelegen. Z. B. wäre es eine sehr lohnende Forschungsperspektive, eine $x$-beliebige Kunstausstellung in allen ihren Erscheinungsformen im Hinblick auf die in und mit ihr nahegelegten und etablierten sozialen Positionen zu rekonstruieren: von der Vorbereitung und Durchführung der Ausstellung selbst, ihrer Vor- und Nachbereitung in den Medien bis hin zu den Gesprächen, die während und in der Ausstellung alltäglich stattfinden. Der vorliegende Beitrag vermag vielleicht dazu ermuntern, dass und wie die Linguistik zu einer solchen Forschungsperspektive beitragen könnte.

16 Die folgende Abbildung ist bewusst unvollständig, was die Ausfüllung der Felder betrifft. Es wurden nur solche Formen berücksichtigt, die im Text zuvor besprochen wurden und bei denen sich eine unmittelbare Beziehung zwischen gewähltem Mittel und der damit konstituierten sozialen Position ergeben. So lässt sich vielleicht auch die Art von Fragen andeuten, die sich hier stellen, z. B.: Wie sehen die für das Bezugnehmen, das Beschreiben und das Deuten typischen Mittel aus, die die Position des KunSTRICHTERS konstituieren? Oder ist es für diese Position gerade konstitutiv, dass diese Aufgaben fast vollständig hinter die Aufgabe des Bewertens zurücktreten?

17 „Der Betrachter ist im Bild“ heißt es z. B. passend bei Kemp (1985). 


\begin{tabular}{|c|c|c|c|c|c|}
\hline $\begin{array}{l}\text { Aufgabe } \\
\text { Soziale } \\
\text { Position }\end{array}$ & $\begin{array}{l}\text { Bezugneh- } \\
\text { men }\end{array}$ & Beschreiben & Deuten & Erläutern & Bewerten \\
\hline $\begin{array}{l}\text { KUNST- } \\
\text { BETRACH- } \\
\text { TER }\end{array}$ & $\begin{array}{l}\text { Zur-Schau- } \\
\text { Stellung } \\
\text { fokussierter } \\
\text { Wahrneh- } \\
\text { mung } \\
\text { (weiß gar nid } \\
\text { was fürn } \\
\text { material das } \\
\text { isch) }\end{array}$ & $\begin{array}{l}\text { Hinweise auf } \\
\text { Abgebildetes } \\
\text { und Sichtbar- } \\
\text { keit (Die Bild- } \\
\text { betrachter } \\
\text { sehen auch } \\
\text { nicht, was die } \\
\text { abgebildeten } \\
\text { Figuren sehen.) }\end{array}$ & $\begin{array}{l}\text { Hinweise } \\
\text { auf Künst- } \\
\text { leragens } \\
\text { (Ist es } \\
\text { erlaubt, in } \\
\text { den hoch } \\
\text { aufgerich- } \\
\text { teten } \\
\text { Haar- } \\
\text { Türmen } \\
\text { der Frau- } \\
\text { en eine } \\
\text { versteckte } \\
\text { und dann } \\
\text { doch } \\
\text { eingestan- } \\
\text { dene } \\
\text { Belusti- } \\
\text { gung des } \\
\text { Malers zu } \\
\text { sehen?) }\end{array}$ & & $\begin{array}{l}\text { Darstellung } \\
\text { von Gefal- } \\
\text { len und } \\
\text { Wirkung / } \\
\text { Eindruck } \\
\text { (unglaub- } \\
\text { lich. einfach } \\
\text { unglaub- } \\
\text { lich) }\end{array}$ \\
\hline $\begin{array}{l}\text { KUNST- } \\
\text { SAMMLER }\end{array}$ & $\begin{array}{l}\text { Identifizieren } \\
\text { (Une Baigna- } \\
\text { de, Asnières) }\end{array}$ & $\begin{array}{l}\text { Hinweise auf } \\
\text { Format und } \\
\text { Material (Öl auf } \\
\text { Leinwand, } 201 \\
x 300 \mathrm{~cm} \text { ) }\end{array}$ & & & \\
\hline $\begin{array}{l}\text { KUNST- } \\
\text { KENNER }\end{array}$ & $\begin{array}{l}\text { Klassifizieren } \\
\text { (Das Werk } \\
\text { Georges } \\
\text { Seurats [...]) }\end{array}$ & & & $\begin{array}{l}\text { Hinweise } \\
\text { auf Motive; } \\
\text { Epoche / } \\
\text { Stil; Tech- } \\
\text { nik; Werk; } \\
\text { historischen } \\
\text { Hintergrund } \\
\text { (Wir sind } \\
\text { jetzt über- } \\
\text { raschend } \\
\text { schnell in } \\
\text { das mögli- } \\
\text { che innere } \\
\text { Zentrum } \\
\text { der Bilder } \\
\text { vorgesto- } \\
\text { Ben. Das } \\
\text { Nicht- } \\
\text { Abgebildete }\end{array}$ & \\
\hline
\end{tabular}




\begin{tabular}{|l|l|l|l|l|l|}
\hline $\begin{array}{l}\text { Aufgabe } \\
\text { Soziale } \\
\text { Position }\end{array}$ & $\begin{array}{l}\text { Bezugneh- } \\
\text { men }\end{array}$ & Beschreiben & Deuten & Erläutern & Bewerten \\
\hline & & & $\begin{array}{l}\text { war das } \\
\text { vorerst } \\
\text { nicht } \\
\text { adäquat } \\
\text { Darstellba- } \\
\text { re. Dazu } \\
\text { bedurfte es } \\
\text { der fortge- } \\
\text { schrittenen } \\
\text { Moderne, } \\
\text { [...]) }\end{array}$ & \\
\hline KUNST- \\
KRITIKER
\end{tabular}

Tab. 2: Kommunikative Aufgaben und soziale Positionierungen

\section{Literatur}

BARTHES, ROLAND (1983): Cy Twombly. Berlin.

BERNHARD, THOMAS (1988): Alte Meister. Frankfurt a. M.

BÖHM, GOTTFRIED (2009): Kaltes Feuer. Figur und Landschaft bei Georges Seurat. In: Zürcher Kunstgesellschaft/Kunsthaus Zürich (Hgg.): Georges Seurat. Figur im Raum. Ostfildern, S. 85-95.

BOURDIEU, PIERRE (1982): Die feinen Unterschiede. Kritik der gesellschaftlichen Urteilskraft. Frankfurt a. M.

Bourdieu, PierRe (1990): Was heißt Sprechen? Die Ökonomie des sprachlichen Tausches. Wien.

BRINKER, KLAUS/ANTOS, GERD/HEINEMANN, WOLFGANG (Hgg.) (2000): Text- und Gesprächslinguistik. Ein internationales Handbuch. Berlin; New York (1. Halbband).

BRINKER, Klaus/Heinemann, WolfGang/SAGER, Sven F. (Hgg.) (2001): Text- und Gesprächslinguistik. Ein internationales Handbuch. Berlin; New York (2. Halbband).

Demand, Christian (2003): Die Beschämung der Philister. Wie die Kunst sich der Kritik entledigte. Springe. 
DEMAND, Christian (2007): Verklärung und Beschämung. Zur Sprache der Kunstkritik. In: HAUSENDORF, HEIKO (Hg.): Vor dem Kunstwerk. Interdisziplinäre Aspekte des Sprechens und Schreibens über Kunst. München, S. 265-281.

DRÖGE, KuRT/HoffMAnN, DetLEF (Hgg.) (2010): Museum revisited. Transdisziplinäre Perspektiven auf eine Institution im Wandel. Bielefeld.

GenAZINO, WilHelm (2009): Herumstehen, Herumsitzen, Herumliegen. Georges Seurats trügerische Idyllen. In: Zürcher Kunstgesellschaft/Kunsthaus Zürich (Hgg.): Georges Seurat. Figur im Raum. Ostfildern, S. 61-67.

HABERMAS, JÜRGEN (2006): Strukturwandel der Öffentlichkeit. Untersuchungen zu einer Kategorie der bürgerlichen Gesellschaft. Mit einem Vorwort zur Neuauflage 1990. Neudruck. Frankfurt a. M.

HAUSENDORF, HEIKO (2000): Zugehörigkeit durch Sprache. Eine linguistische Studie am Beispiel der deutschen Wiedervereinigung. Tübingen.

HAUSENDORF, Heiko (2003): Deixis and Speech Situation Revisited. The Mechanism of Perceived Perception. In: LENZ, Friedrich (Hg.): Deictic Conceptualisiation of Space, Time and Person. Amsterdam, S. 249-269.

HAUSENDORF, HEIKO (2005): Die Kunst des Sprechens über Kunst. Zur Linguistik einer riskanten Kommunikationspraxis. In: Klotz, Peter/Lubkoll, Christine (Hgg.): Beschreibend wahrnehmen - wahrnehmend beschreiben. Sprachliche und ästhetische Aspekte kognitiver Prozesse. Freiburg i. Br., S. 99-134.

HAUSENDORF, HeIKO (2006): Gibt es eine Sprache der Kunstkommunikation? Linguistische Zugangsweisen zu einer interdisziplinären Thematik. In: Paragrana. Internationale Zeitschrift für Historische Anthropologie 15/2 (2006), S. 65-98.

HAUSENDORF, HEIKO (2007a): Die Sprache der Kunstkommunikation und ihre interdisziplinäre Relevanz. In: HAUSENDORF, HEIKO (Hg.): Vor dem Kunstwerk. Interdisziplinäre Aspekte des Sprechens und Schreibens über Kunst. München, S. 17-51.

HAUSENDORF, HeiKo (Hg.) (2007b): Vor dem Kunstwerk. Interdisziplinäre Aspekte des Sprechens und Schreibens über Kunst. München.

HAusENDORF, Heiko/BorA, Alfons (2006): Reconstructing Social Positioning in Discourse: Methodological Basics and their Implementation from a Conversation Analysis Perspective. In: Hausendorf, HeIKo/Bora, Alfons (Hgg.): Analysing Citizenship Talk. Social Positioning in Political and Legal Decision-making Processes. Amsterdam, S. 85-97.

Heath, Christian/Vom Lehn, DiRK (2004): Configuring Reception. (Dis-)Regarding the ,Spectator ' in Museums and Galleries. In: Theory, Culture \& Society 21/6 (2004), S. 43-65.

Huber, Hans Dieter/LOCher, Hubert/Schulte, Karin (Hgg.) (2002): Kunst des Ausstellens. Beiträge, Statements, Diskussionen. Staatliche Akademie der Bildenden Künste Stuttgart. Ostfildern-Ruit.

KemP, WolfGANG (1985): Der Betrachter ist im Bild. Kunstwissenschaft und Rezeptionsästhetik. Köln.

KINDT, WALTHER (1982): Social Functions of Communication about Works of Art. In: Poetics 11 (1982), S. 393-418.

Kravagna, Christian (Hg.) (2001): Das Museum als Arena. Institutionskritische Texte von KünstlerInnen. Köln.

LOCHER, HUBERT (2002): Die Kunst des Ausstellens. Anmerkungen zu einem unübersichtlichen Diskurs. In: Huber, HANS Dieter /LOCHER, Hubert/Schulte, KARIN (Hgg.): Kunst des Ausstellens. Beiträge, Statements, Diskussionen. Staatliche Akademie der Bildenden Künste Stuttgart. Ostfildern-Ruit, S. 14-30.

LÜDDEMANN, STEFAN (2007): Mit Kunst kommunizieren. Theorien, Strategien, Fallbeispiele. Wiesbaden.

LuHMANN, NiKLAS (1997): Die Kunst der Gesellschaft. Frankfurt a. M.

O’DOHERTY, BRIAN (1996): In der weißen Zelle. Inside the White Cube. Berlin. 
OEVERMANN, UlRICH (1996): Krise und Muße. Struktureigenschaften ästhetischer Erfahrung aus soziologischer Sicht. Vortrag am 19.06.1996 in der Städel-Schule. (Online verfügbar unter:www.w-f-.de/PDFDateien/Ulrich\%20Oevermann,\%20Krise\%20und\%20Musse.pdf.)

PolenZ, PETER vON (1988): Deutsche Satzsemantik. Grundbegriffe des Zwischen-den-ZeilenLesens. 2. Aufl. Berlin; New York.

Smolik, Noemi (2001): Sprache als Tarnung. Zum Stand der heutigen Kunstkritik. In: VITT, WALTER (Hg.): Vom Kunststück, über Kunst zu schreiben. 50 Jahre AICA Deutschland. Nördlingen, S. 101-107.

Thim-Mabrey, Christiane (2001): Grenzen der Sprache - Möglichkeiten der Sprache. Untersuchungen zur Textsorte Musikkritik. Frankfurt a. M.

Thim-MABrey, Christiane (2007): Linguistische Aspekte der Kommunikation über Kunst. In: HAUSENDORF, HEIKO (Hg.): Vor dem Kunstwerk. Interdisziplinäre Aspekte des Sprechens und Schreibens über Kunst. München, S. 99-121.

UlLRICH, WOLFGANG (2003): Tiefer hängen. Über den Umgang mit der Kunst. Berlin.

UlLrich, WolfGang (2007): „Ein bisschen dumm“ - die Rollen des Kunstrezipienten. In: HAU SENDORF, HEIKO (Hg.): Vor dem Kunstwerk. Interdisziplinäre Aspekte des Sprechens und Schreibens über Kunst. München, S. 197-222.

Vogt, ToBias (2006): Untitled. Zur Karriere unbetitelter Kunst in der jüngsten Moderne. München.

Vom Lehn, Dirk/Heath, Christian (2007): Perspektiven der Kunst - Kunst der Perspektiven. In: HAUSENDORF, HeIKo (Hgg.): Vor dem Kunstwerk. Interdisziplinäre Aspekte des Sprechens und Schreibens über Kunst. München, S. 147-170.

WulfFEN, ThOMAS (Hg.) (1994): Betriebssystem Kunst. Eine Retrospektive. In: Kunstforum 125 (1994), S. 49-58.

ZIESE, MAREN (2010): Kuratoren und Besucher. Modelle kuratorischer Praxis in Kunstausstellungen. Bielefeld. 